\title{
Towards an Accurate and Robust Roe-Type Scheme for All Mach Number Flows
}

\author{
Wenjia Xie*, Ye Zhang, Qing Chang and Hua Li \\ College of Aerospace Science and Engineering, National University of Defense \\ Technology, Changsha 410073, Hunan, China
}

Received 09 June 2018; Accepted (in revised version) 14 October 2018

\begin{abstract}
We propose an accurate and robust Roe-type scheme applied to the compressible Euler system at all Mach numbers. To study the occurrence of unstable modes during the shock wave computation, a shock instability analysis of several Roe-type schemes is carried out. This analysis approach allows to propose a simple and effective modification to eliminate shock instability of the Roe method for hypersonic flows. A desirable feature of this modification is that it does not resort to any additional numerical dissipation on linear degenerate waves to suppress the shock instability. With an all Mach correction strategy, the modified Roe-type scheme is further extended to solve flow problems at all Mach numbers. Numerical results that are obtained for various test cases indicate that the new scheme has a good performance in terms of accuracy and robustness.
\end{abstract}

AMS subject classifications: 35L65, 65M08, 76M12, 76L05

Key words: Roe scheme, low Mach number, numerical shock instability.

\section{Introduction}

Due to their physical background, Godunov-type schemes become one of the most effective techniques for shock-capturing. They play a fundamental role in modern CFD methods for compressible flows at moderate Mach numbers. However, it has been well demonstrated that Godunov-type schemes are not always reliable in cases where the flow speed is hypersonic or the Mach number tends to zero. The high speed flow problems usually involve complex flow phenomena, such as strong shock waves, shockvortex interactions, shock-boundary layer interactions and shear layers. Prediction of these problems requires robust, efficient and accurate numerical methods. Unfortunately, Godunov-type schemes that involve minimal dissipation on linear degenerated waves

*Corresponding author.

Emails: xiewenjia@nudt.edu.cn (W. J. Xie), zhangye17a@nudt.edu.cn (Y. Zhang), changqing16@nudt.edu.cn (Q. Chang), lihuakd@tom.com (H. Li) 
are vulnerable to shock instability problems, including the carbuncle phenomenon [1]. In [2], the authors present a wide-ranging survey of numerical experiments on a large collection of flux functions for hypersonic flow computations, the results have demonstrated that none of the tested schemes is able to balance the low dissipation and the high robustness well at the same time, all the tested solvers exhibit numerical shock instabilities to some extent. The reliability of these methods will become even worse for hypersonic flow computations in cases where the computational grid is complex [3] or the schemes are extended to their higher order versions [4]. Meanwhile, it is common that compressible and weakly compressible flow regimes coexist in the domain of interest. Even in high speed flows, there are still low Mach number regions such as boundary layers, stagnation regions and the wake behind bodies. Special attention should also be paid to the accuracy of numerical methods at low speeds, for example, the aerodynamic heating predictions [5,6] and the turbulence flow simulations [7]. This explains the need for solvers that are able to deal with problems where the flow regime may vary from low to high Mach values.

The Roe scheme [8] is perhaps one of the most famous approximate Riemann solvers. Due to its good performance for moderate Mach number flows, the Roe scheme has been widely studied and applied to simulations of engineering problems. However, the original Roe method has several shortcomings, for example, the violation of the entropy condition and non-positivity in low density flows. These deficiencies have been fixed properly [9-11], but there is still a challenging problem that has not been well explored or clearly understood. As other low-diffusion flux functions, the Roe approach also suffers from shock anomalies at strong shock cases such as the carbuncle phenomenon, which significantly reduces its reliability in hypersonic flow computations. Since its first discovery by Peery and Imaly [12], the carbuncle phenomenon has been widely studied by researchers and engineers. A large variety of work has been devoted to understanding and curing this problem. Readers are suggested to refer to [1,13-15] and the references therein for detailed reviews of this problem. We remark that there is still no a clear consensus on the mechanism of the carbuncle phenomenon. Quirk [16] may be the first to systematically analyze the unfavorable pathologies of Godunov-type schemes for flows at high Mach numbers. He finds that dissipative numerical fluxes, which smear entropy waves and shear waves severely, are endowed with high resistance against shock anomalies. To cure the shock instability of the Roe scheme, he suggests to combine it with a more dissipative HLLE scheme [17]. Following Quirk, many works are devoted to improving the robustness of the Roe method by introducing additional numerical or physical dissipation. These modifications include the hybrid technique which combines two different schemes with different dissipative properties $[16,18]$, the entropy fix technique that includes additional dissipation via modifying eigenvalues of the related dissipative matrix [19-21] and the rotated Riemann solver approach that introduces more numerical dissipation by a rotated manner [22-24]. These methods are all effective in suppressing shock instabilities of numerical methods at strong shocks. However, although these modifications are implemented by different strategies, they usually rely on certain switching 
function to control the numerical dissipation. To avoid compromising the accuracy of the numerical method, special attention should be paid to the switching function used in the hybrid strategy, especially for unstructured grids. For example, Zhang et al. [24] find that the rotated strategy, which helps improve the robustness of low diffusive schemes against strong shocks, will introduce additional numerical dissipation for viscous flows. To cure this deficiency, a pressure-weighted rotation strategy is proposed. In [25], the authors use the hybrid technique to improve the shock stability of SLAU scheme [26] on unstructured grids. Believing that the momentum perturbation in numerical fluxes is the main source of shock instability, the authors only apply the hybrid dissipation terms on the momentum fluxes of the numerical flux function with the help of a multidimensional pressureweighted function. In some circumstances, it can not be guaranteed that these additional dissipation will not influence the high resolution of the original solver especially in cases where complex flow patterns are involved. Thus, how to cure the carbuncle phenomena of Godunov-type schemes without resorting to additional dissipation becomes an attractive issue. In our previous work [14], we found two useful conclusions concerning the shock instability of Godunov-type schemes. By a shock instability analysis of a simplified carbuncle problem (i.e., a stationary normal shock problem in two dimensions), we are able to clarify that the perturbed errors that trigger the shock instability originate from the numerical shock structure. Moreover, it was found that the shock instability would be suppressed if the mass flux across the normal shock was correctly preserved. Such conclusions allow to clarify the connection between the numerical dissipation and the shock instability. That is, the added numerical dissipation will damp perturbed errors generated inside the numerical shock structure and help maintain the correct mass flux across the normal shock. However, in the current study, we will demonstrate that these conclusions can also be used to help propose cures for the shock instability without introducing additional numerical dissipation on linear degenerate waves. Furthermore, by a shock instability analysis of the Roe-type schemes, we can also justify a modified Roe-type scheme, i.e., RoeM [28] that is endowed with improved resistance against shock anomalies. The RoeM scheme proposed by Kim et al. [28] features several desirable properties such as the entropy satisfaction property, the robustness in low density flows. It is also able to suppress the shock instability at least to some extent. Contrasting to other modified Roe-type schemes, the RoeM approach is able to improve the robustness of the numerical method without including additional numerical dissipation on linear degenerate waves. The authors base their modification on Liou's conjecture [29] which argues that the root of causing the shock instability is the pressure difference in the dissipative term that appears in the numerical evaluation of convective fluxes. However, it has been found that Liou's conjecture does not identify correctly the source of the numerical shock instability $[30,31]$. That is may be the reason why the RoeM solver also produces the carbuncle phenomenon in certain cases. In the current study, we will demonstrate that the robustness of the RoeM scheme can be enhanced without compromising its high resolution and desirable features.

Another deficiency of the Roe scheme is the lack of accuracy in the low Mach 
regime [32]. As other Godunov-type schemes, the Roe solver may generate spurious numerical diffusion in simulations of low Mach flows in spite of its good performance at capturing shocks. The accuracy problem of Godunov-type schemes in low Mach regime has been widely investigated in the literature, see [32-36] and the references therein. In order to capture the low Mach limit, several modifications are proposed to recover a proper convergence toward the incompressible limit. They include, but are not limited to the preconditioned methods [32,39], the all-speed scheme [35,40,41] and the Rieper's fix [34,42]. Herein, we have a particular interest in the all Mach correction method proposed by Dellacherie et al. [36,43]. Different from other common cures that are specified to certain numerical schemes, the all Mach correction method is general and can be applied to many numerical schemes more than the Godunov-type ones. Although these modifications may rely on different arguments, the main ingredient of these techniques will usually be to modify the artificial viscosity in the momentum equation for low Mach number values [44]. However, there is still one noteworthy problem with these all Mach schemes. They usually cannot avoid numerical instabilities near strong shocks in hypersonic flow regime. Some work also concerns the shock instabilities of low Mach schemes, for example, the low-Mach Roe-type scheme proposed in [42], but the method also resorts to additional numerical dissipation on linear degenerate waves to avoid shock instabilities. When it comes to a true all Mach Godunov-type scheme, the flux function should not only maintain its robustness at fairly high Mach numbers without compromising its high resolution of different waves, but also produces accurate solutions at low Mach numbers. That is what we are striving for in the current study.

The outline of the rest of this paper is as follows. In Section 2, governing equations of compressible flows and their related finite volume discretization are presented. Several Roe-type schemes are also reviewed in the same section. In Section 3, we conduct a stability analysis of the Roe-type methods. A shock-stable enhanced Roe-type scheme is also given. In Section 4, we extend the Roe-type scheme to simulate flows at low Mach numbers. The accuracy and robustness of the proposed method are tested in Section 5. Finally, concluding remarks are made in Section 6.

\section{Governing equations and discretization}

We first consider the two-dimensional Euler equations that model inviscid, compressible flows

$$
\frac{\partial \mathbf{U}}{\partial t}+\frac{\partial \mathbf{F}(\mathbf{U})}{\partial x}+\frac{\partial \mathbf{G}(\mathbf{U})}{\partial y}=0,
$$

where $\mathbf{U}$ is the vector of conservative variables, $\mathbf{F}(\mathbf{U})$ and $\mathbf{G}(\mathbf{U})$ are the vectors of conservative fluxes, they are defined as 


$$
\mathbf{U}=\left(\begin{array}{c}
\rho \\
\rho u \\
\rho v \\
E
\end{array}\right), \quad \mathbf{F}(\mathbf{U})=\left(\begin{array}{c}
\rho u \\
\rho u^{2}+p \\
\rho u v \\
u(E+p)
\end{array}\right), \quad \mathbf{G}(\mathbf{U})=\left(\begin{array}{c}
\rho v \\
\rho u v \\
\rho v^{2}+p \\
v(E+p)
\end{array}\right),
$$

where $\rho$ is the density, $p$ is the pressure, $\mathbf{u}=(u, v)$ is the flow velocity, $E$ is the total energy per unit volume. This set of equations is closed using the perfect gas law, for which the equation of state is in the form

$$
p=(\gamma-1)\left[E-\frac{1}{2} \rho\left(u^{2}+v^{2}\right)\right]
$$

with $\gamma=1.4$ being the specific heat ratio.

We consider solving the system (2.1) numerically in the framework of a structured cell-centered finite-volume method

$$
\frac{d \mathbf{U}_{i}}{d t}+\frac{1}{\left|\Omega_{i}\right|} \sum_{\Gamma_{i j} \subset \partial \Omega_{i}}\left|\Gamma_{i j}\right| \boldsymbol{\Phi}_{i j}=0 .
$$

In Eq. (2.4), it is assumed that the domain $\Omega$ is discretized by some structured quadrilateral cells $\Omega_{i}$. The volume is denoted by $\left|\Omega_{i}\right| . \Gamma_{i j}$ denotes the common edge of two neighboring cells $\Omega_{i}$ and $\Omega_{j}$ and $\mathbf{n}_{i j}$ represents the unit vector normal to $\Gamma_{i j}$ pointing from $\Omega_{i}$ to $\Omega_{j}$, i.e., $\mathbf{n}_{i j}=\left(n_{x}, n_{y}\right)_{i j} . \boldsymbol{\Phi}_{i j}$ is a Roe-type scheme, which is a numerical approximation to the normal flux $\mathbf{H}_{n_{i j}}=[\mathbf{F}, \mathbf{G}] \cdot \mathbf{n}_{i j}$ at the interface.

\subsection{Roe-Pike method}

Since its first presentation, the Roe scheme [8] has been studied extensively and also applied to a large variety of physical problems. Whereas, the original Roe approach requires explicitly the computation of the Roe averaged Jacobian matrix, which is not easy to deal with for complicated sets of hyperbolic conservation laws. To simplify the Roe scheme, Roe and Pike [45] made refinements to the original approach. The numerical flux of Roe-Pike method is used as an approximation to the normal flux at the interface between the constant left and right states. It can be written as follows:

$$
\boldsymbol{\Phi}_{\text {Roe }}=\frac{1}{2}\left[\mathbf{H}_{n}\left(\mathbf{U}_{L}\right)+\mathbf{H}_{n}\left(\mathbf{U}_{R}\right)\right]-\frac{1}{2} \sum_{i=1}^{4} \widehat{\alpha}_{i}\left|\widehat{\lambda}_{i}\right| \widehat{\mathbf{R}}_{i},
$$

where $\mathbf{H}_{n}\left(\mathbf{U}_{L / R}\right)$ denotes the left or the right normal flux at the interface (the subscript $i j$ of the unit vector is omitted for clarity). $\widehat{\alpha}_{i}$ represent the wave strengths, $\widehat{\lambda}_{i}$ are the 
eigenvalues and $\widehat{\mathbf{R}}_{i}$ are the right eigenvectors, they are given by

$$
\begin{array}{llll}
\widehat{\alpha}_{1}=\frac{\Delta p-\widehat{\rho} \widehat{a} \Delta q_{n}}{2 \widehat{a}^{2}}, & \widehat{\alpha}_{2}=\Delta \rho-\frac{\Delta p}{\widehat{a}^{2}}, & \widehat{\alpha}_{3}=\widehat{\rho} \Delta q_{t}, & \widehat{\alpha}_{4}=\frac{\Delta p+\widehat{\rho} \widehat{a} \Delta q_{n}}{2 \widehat{a}^{2}}, \\
\widehat{\lambda}_{1}=\widehat{q}_{n}-\widehat{a}, & \widehat{\lambda}_{2}=\widehat{q}_{n}, & \widehat{\lambda}_{3}=\widehat{q}_{n}, & \widehat{\lambda}_{4}=\widehat{q}_{n}+\widehat{a}, \\
\widehat{\mathbf{R}}_{1}=\left(\begin{array}{c}
1 \\
\widehat{u}-\widehat{a} n_{x} \\
\widehat{v}-\widehat{a} n_{y} \\
\widehat{H}-\widehat{a} \widehat{q}_{n}
\end{array}\right), & \widehat{\mathbf{R}}_{2}=\left(\begin{array}{c}
1 \\
\widehat{u} \\
\widehat{v} \\
\frac{1}{2}\left|\widehat{q}_{n}\right|^{2}
\end{array}\right), & \widehat{\mathbf{R}}_{3}=\left(\begin{array}{c}
0 \\
-n_{y} \\
n_{x} \\
\widehat{q}_{t}
\end{array}\right), & \widehat{\mathbf{R}}_{4}=\left(\begin{array}{c}
1 \\
\widehat{u}+\widehat{a} n_{x} \\
\widehat{v}+\widehat{a} n_{y} \\
\widehat{H}+\widehat{a} \widehat{q}_{n}
\end{array}\right) .
\end{array}
$$

Herein, $\widehat{(\cdot)}$ denote the Roe averaged values, $q_{n}=(u, v) \cdot \mathbf{n}$ is the velocity component in the normal direction $\mathbf{n}=\left(n_{x}, n_{y}\right), q_{t}=(u, v) \cdot\left(-n_{y}, n_{x}\right)$ denotes the velocity component in the tangential direction. $a$ denotes the speed of sound and $\Delta$ represents the difference between the right and left quantities, i.e., $\Delta(\cdot)=(\cdot)_{R}-(\cdot)_{L}$.

\subsection{Roe scheme in an alternative form}

The Roe scheme can also be further rewritten into an alternative form that is much simpler. Einfeldt [17] showed that the Roe scheme could be considered as a HLL-type method with an anti-diffusion term in the linear degenerate fields. The methodology was followed by Kim et al. [28] to develop a shock-stable enhanced Roe solver. The new formulation of the Roe scheme does not require the full computation of the right eigenvectors and the Roe linearization approach. Thus, extension to more general nonlinear systems of conservation laws can be implemented in a simpler way. The alternative Roe scheme can be written as

$$
\boldsymbol{\Phi}_{\text {Roe }}=\frac{S_{R}^{+} \mathbf{H}_{n}\left(\mathbf{U}_{L}\right)-S_{L}^{-} \mathbf{H}_{n}\left(\mathbf{U}_{R}\right)}{S_{R}^{+}-S_{L}^{-}}+\frac{S_{L}^{-} S_{R}^{+}}{S_{R}^{+}-S_{L}^{-}} \Delta \mathbf{U}-\frac{S_{L}^{-} S_{R}^{+}}{S_{R}^{+}-S_{L}^{-}} \frac{1}{1+|\widehat{M}|} \mathbf{B} \Delta \mathbf{U},
$$

where $\Delta \mathbf{U}$ denotes the difference between the right and left conservative variables, $\mathbf{B} \Delta \mathbf{U}$ represents the compact form of the summation $\widehat{\alpha}_{2} \widehat{\mathbf{R}}_{2}+\widehat{\alpha}_{3} \widehat{\mathbf{R}}_{3}$, they are expressed as follows:

$$
\Delta \mathbf{U}=\Delta\left(\begin{array}{c}
\rho \\
\rho u \\
\rho v \\
E
\end{array}\right), \quad \mathbf{B} \Delta \mathbf{U}=\left(\Delta \rho-\frac{\Delta p}{\widehat{a}^{2}}\right)\left(\begin{array}{c}
1 \\
\widehat{u} \\
\widehat{v} \\
\frac{1}{2}\left|\widehat{q}_{n}\right|^{2}
\end{array}\right)+\widehat{\rho}\left(\begin{array}{c}
0 \\
\Delta u-\Delta \widehat{q}_{n} n_{x} \\
\Delta v-\Delta \widehat{q}_{n} n_{y} \\
\widehat{u} \Delta u+\widehat{v} \Delta v-\widehat{q}_{n} \Delta q_{n}
\end{array}\right)
$$

the numerical wave speeds $S_{L}^{-}$and $S_{R}^{+}$are approximations of the physical wave speeds from the Riemann problem at the cell interface. For the Roe scheme, they are defined as

$$
S_{L}^{-}=\min \left(0, \quad \widehat{q}_{n}-\widehat{a}\right), \quad S_{R}^{+}=\max \left(0, \quad \widehat{q}_{n}+\widehat{a}\right) .
$$




\subsection{RoeM scheme}

The Roe scheme defined in (2.5) or (2.7) is known to suffer from several nonphysical problems, such as the violation of the entropy condition, failure to preserve positivity and numerical shock anomalies with strong shocks $[9,10,16]$. To cure these numerical defects, Kim et al. [28] proposed a modified version of the Roe scheme in the form of the numerical flux function defined in (2.7). The resulting scheme denoted as RoeM can be written as follows:

$$
\boldsymbol{\Phi}_{\mathrm{RoeM}}=\frac{S_{R}^{+} \mathbf{H}_{n}\left(\mathbf{U}_{L}\right)-S_{L}^{-} \mathbf{H}_{n}\left(\mathbf{U}_{R}\right)}{S_{R}^{+}-S_{L}^{-}}+\frac{S_{L}^{-} S_{R}^{+}}{S_{R}^{+}-S_{L}^{-}} \Delta \mathbf{U}^{*}-\frac{S_{L}^{-} S_{R}^{+}}{S_{R}^{+}-S_{L}^{-}} \frac{1}{1+|\widehat{M}|} \mathbf{B} \Delta \mathbf{U},
$$

with

$$
\Delta \mathbf{U}^{*}=\Delta\left(\begin{array}{c}
\rho \\
\rho u \\
\rho v \\
\rho H
\end{array}\right), \quad \mathbf{B} \Delta \mathbf{U}=\left(\Delta \rho-f_{\operatorname{RoeM}} \frac{\Delta p}{\widehat{a}^{2}}\right)\left(\begin{array}{c}
1 \\
\widehat{u} \\
\widehat{v} \\
\widehat{H}
\end{array}\right)+\widehat{\rho}\left(\begin{array}{c}
0 \\
\Delta u-\Delta q_{n} n_{x} \\
\Delta v-\Delta q_{n} n_{y} \\
\Delta H
\end{array}\right),
$$

where the function $f_{\text {Roem }}$ is used to reduce the rate at which pressure perturbation feeds density field, which is claimed to be effective in improving the robustness of the Roe scheme. The function $f_{\text {RoeM }}$ is defined as

$$
f_{\text {RoeM }}= \begin{cases}1, & \widehat{u}^{2}+\widehat{v}^{2}=0, \\ |\widehat{M}|^{h}, & \text { otherwise, }\end{cases}
$$

with

$$
h=1-\min \left(p_{i, j+1 / 2}, p_{i-1 / 2, j}, p_{i+1 / 2, j}, p_{i-1 / 2, j+1}, p_{i+1 / 2, j+1}\right),
$$

and

$$
p_{i, j+1 / 2}=\min \left(p_{i, j} / p_{i, j+1}, p_{i, j+1} / p_{i, j}\right) .
$$

It should be noted that the conservative variable in the energy equation has been modified to preserve total enthalpy. For the RoeM approach defined in (2.10)-(2.14), the following wave speeds estimate is used

$$
S_{L}^{-}=\min \left(0, \widehat{q}_{n}-\widehat{a}, \quad\left(q_{n}\right)_{L}-a_{L}\right), \quad S_{R}^{+}=\max \left(0, \widehat{q}_{n}+\widehat{a}, \quad\left(q_{n}\right)_{R}+a_{R}\right) .
$$

It has been argued in [17] that the above estimate introduces the least amount of numerical dissipation for isolated shocks and can introduce enough numerical dissipation to avoid the occurrence of the nonphysical expansion shock. In some circumstance, the wave speeds estimate also helps preserve positivity. A simpler but more diffusive estimate [27] can also be applied

$$
S_{L}^{-}=\min \left(0, \quad\left(q_{n}\right)_{L}-a_{L}, \quad\left(q_{n}\right)_{R}-a_{R}\right), \quad S_{R}^{+}=\max \left(0, \quad\left(q_{n}\right)_{L}+a_{L}, \quad\left(q_{n}\right)_{R}+a_{R}\right) .
$$


The RoeM method is a desirable modified version of the Roe scheme, because it eliminates most of the defects of the original Roe approach including the violation of the entropy condition, the non-positivity and the shock anomalies in some circumstance. Meanwhile, it still preserves the high resolution of all kinds of discontinuities as the original Roe scheme does. However, we have found that the RoeM method still exhibits some numerical shock anomalies in the vicinity of strong shocks. The limited robustness of the RoeM scheme at strong shocks can be attributed to the unreliable explanation of the cause of shock instability. To further enhance the robustness of the RoeM scheme at strong shocks, the authors in [28] propose another modified version of the Roe scheme, i.e., RoeM2, it is written by

$$
\boldsymbol{\Phi}_{\mathrm{RoeM} 2}=\frac{S_{R}^{+} \mathbf{H}_{n}\left(\mathbf{U}_{L}\right)-S_{L}^{-} \mathbf{H}_{n}\left(\mathbf{U}_{R}\right)}{S_{R}^{+}-S_{L}^{-}}+\frac{S_{L}^{-} S_{R}^{+}}{S_{R}^{+}-S_{L}^{-}} \Delta \mathbf{U}^{*}-g \frac{S_{L}^{-} S_{R}^{+}}{S_{R}^{+}-S_{L}^{-}} \frac{1}{1+|\widehat{M}|} \mathbf{B} \Delta \mathbf{U},
$$

where the function $g$ can be defined as

$$
g= \begin{cases}|\widehat{M}|^{1-\min \left(\frac{p_{j}}{p_{j+1}}, \frac{p_{j+1}}{p_{j}}\right),}, \widehat{M} \neq 0, \\ 1, & \widehat{M}=0 .\end{cases}
$$

It can be observed that the function $g$ that appears at interfaces normal to the shock will become a small value, thus the dissipative term $\mathbf{B} \Delta \mathbf{U}$ corresponding to contact and shear waves will be reduced. As a result, the flux function defined in Eqs. (2.17) and (2.18) will approach the HLLE solver at shocks. The improved robustness of the RoeM2 scheme is due to the introduced additional multidimensional numerical dissipation corresponding to the linear degenerate waves. In contrast, the technique used in RoeM scheme does not include such numerical dissipation. In the next section, we will conduct a shock instability analysis of these Roe-type schemes. This analysis approach allows to propose a more reliable and robust Roe-type method without introducing any additional numerical dissipation on linear degenerate waves.

\section{A shock instability analysis}

In this section, we make efforts to improve the robustness of the Roe method for capturing strong shocks in hypersonic flows. Contrasting to other common cures for the shock instability such as the hybrid techniques $[16,23]$ or entropy fix methods $[12,20,46]$, the proposed cure for the Roe solver does not depend on additional numerical dissipation on linear degenerate waves. Thus, the high resolution of the original Roe flux is preserved. To carry out the numerical analysis of the shock stability, we start with a simplified shock instability problem. 


\subsection{A simplified shock instability problem}

Numerical flux functions with minimal dissipation on linear degenerate waves are known to produce shock anomalies for high Mach number flows. To explore the cause and analyze the behaviors of shock instability, it is wise to resort to a simplified problem which preserves the essence of shock instability. The numerical test problem considered here is the stationary normal shock wave problem in a two-dimensional rectangular domain. This test case, which was proposed firstly by Sanders et al. [21] and modified by Dumbser et al. [30] and Kitamura et al. [2], has been widely used to evaluate the robustness of various shock-capturing schemes. Here, we repeat the setup of this numerical test for self-containedness. Readers are referred to references $[2,14]$ for the more detailed description.

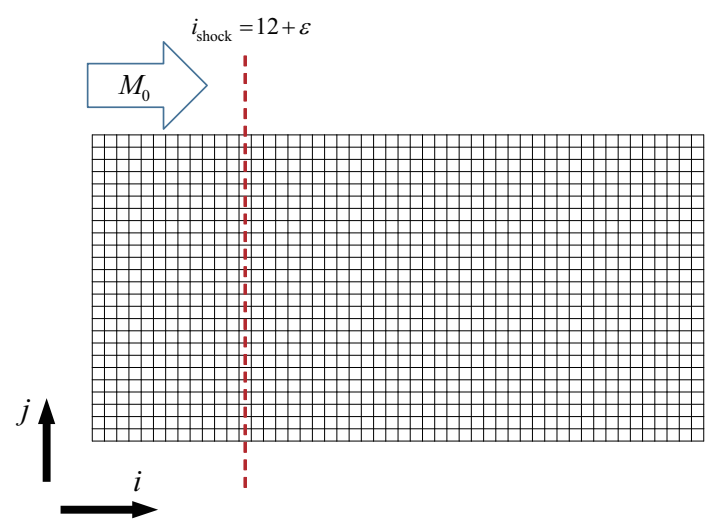

Figure 1: Computational grid and conditions for Kitamura's steady normal shock test.

As shown in Fig. 1, the computational domain is discretized into $50 \times 25$ uniform cells without perturbations. The initial conditions are prescribed for left $(L: i \leq 12)$ and right $(R: i \geq 14)$ following the Rankine-Hugoniot conditions across the normal shock as

$$
\mathbf{U}_{L}=\left(\begin{array}{c}
1 \\
1 \\
0 \\
\frac{1}{\gamma(\gamma-1) M_{0}^{2}}+\frac{1}{2}
\end{array}\right), \quad \mathbf{U}_{R}=\left(\begin{array}{c}
f\left(M_{0}\right) \\
1 \\
0 \\
\frac{g\left(M_{0}\right)}{\gamma(\gamma-1) M_{0}^{2}}+\frac{1}{2 f\left(M_{0}\right)}
\end{array}\right)
$$

where

$$
f\left(M_{0}\right)=\left(\frac{2}{(\gamma+1) M_{0}^{2}}+\frac{\gamma-1}{\gamma+1}\right)^{-1}, \quad g\left(M_{0}\right)=\frac{2 \gamma M_{0}^{2}}{\gamma+1}-\frac{\gamma-1}{\gamma+1} .
$$

The freestream Mach number is set as $M_{0}=6.0$ which is a critical value of the carbuncle for ideal gases [47]. The Roe scheme captures the shock with only one-internal point that belongs to a Hugoniot curve. The internal shock conditions $(M: i=13)$ are set as 
intermediate states taken from the Hugoniot curve, as in [48]:

$$
\begin{aligned}
& \rho_{M}=\left(1-\alpha_{\rho}\right) \rho_{L}+\alpha_{\rho} \rho_{R}, \\
& u_{M}=\left(1-\alpha_{u}\right) u_{L}+\alpha_{u} u_{R}, \\
& p_{M}=\left(1-\alpha_{p}\right) p_{L}+\alpha_{p} p_{R},
\end{aligned}
$$

where

$$
\begin{aligned}
& \alpha_{\rho}=\varepsilon, \\
& \alpha_{u}=1-(1-\varepsilon)\left(1+\varepsilon \frac{M_{0}^{2}-1}{1+(\gamma-1) M_{0}^{2} / 2}\right)^{-1 / 2}\left(1+\varepsilon \frac{M_{0}^{2}-1}{1-2 \gamma M_{0}^{2} /(\gamma-1)}\right)^{-1 / 2}, \\
& \alpha_{p}=\varepsilon\left(1+(1-\varepsilon) \frac{\gamma+1}{\gamma-1} \frac{M_{0}^{2}-1}{M_{0}^{2}}\right)^{-1 / 2} .
\end{aligned}
$$

The inflow boundary conditions are set to freestream values. According to [49], the mass flux at the ghost cell of the outflow boundary is prescribed as

$$
(\rho u)_{i \max +1, j}=(\rho u)_{0}=1
$$

in order for the mass in the whole computational domain to remain constant and to fix the shock at the same position. Meanwhile, other values are simply extrapolated. The periodical condition is imposed on the boundaries of $j$ direction. The computations are conducted for 80,000 steps with $C F L=0.5$. If a scheme is able to produce stable and converged solutions for all the shock positions $\varepsilon$, the scheme can be considered as shockstable for this test.

\subsection{Cures for the shock instability of Roe-type schemes}

\subsubsection{Cause of the shock instability}

To propose a reliable cure for the shock instability of the Roe-type scheme, we need to know the underling mechanism of the occurrence of unstable modes during the shock wave computation. Unfortunately, there is still no general consensus on some facets of this complicated problem and the issue is still actively debated. In our previous study [14], we found two useful conclusions that may be helpful to further the understanding of the shock instability of Godunov-type schemes. By carrying out numerical stability analysis of several Godunov-type schemes through systematic numerical experiments and a linear perturbation analysis, we are able to clarify that the spatial location of the shock instability originates from the intermediate states inside the shock structure. Furthermore, it is demonstrated that if the mass flux across the normal shock is correctly preserved during the computation, then the shock wave can be captured stably. In Fig. 2, we plot the diagram of the numerical shock structure. As shown, when the instability happens, the perturbed errors will be generated inside the cell $M$, then they will be 


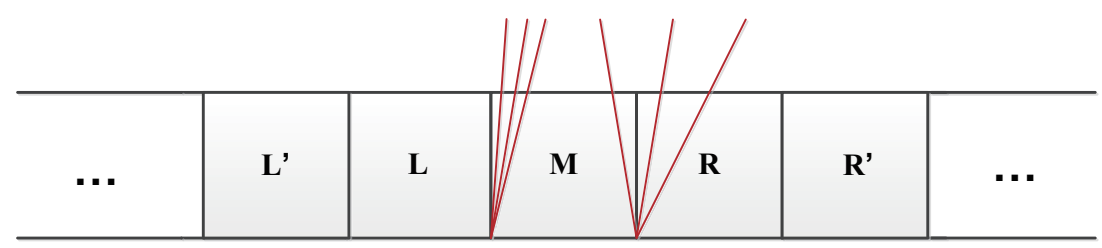

Figure 2: Numerical shock structure and wave patterns.

transported downstream on right-going waves. The perturbed errors will lead to erroneous mass flux in cell $R$, i.e., $(\rho u)_{L} \neq(\rho u)_{R}$, which is considered to be the cause of the shock instability. We remark that the mentioned two conclusions have been validated through numerical experiments and further justified by a perturbation analysis. Also, they pave ways to suppressing the shock instability of Godunov-type schemes for strong shocks. For example, the robustness of numerical schemes can be improved by introducing multidimensional dissipation to damp perturbation errors inside the numerical shock structure and then help maintain the correct mass flux across the normal shock. Such a strategy is commonly used to cure shock instabilities by hybrid flux functions. Alternatively, we can control the transportation of the erroneous perturbations from the intermediate states to the downstream cells. The later technique has an advantage over the former one because it does not introduce additional multidimensional dissipation on shear waves to enhance the stability. Next, we will demonstrate that the robustness of the Roe method can be improved by controlling the perturbed errors that are transported from the intermediate states inside the shock structure to the downstream regions.

\subsubsection{A linear perturbation analysis}

In this section, we carry out a linear perturbation analysis of the Roe-type schemes presented in Eqs. (2.7) and (2.10). This method was first used by Quirk [16] and followed by the authors in $[14,28,50,51]$ to explore the mechanism of shock instability. With the perturbation analysis method, it is convenient to study the unstable behaviors of the perturbed errors and their propagation. Here, we combine this analysis method with the two useful conclusions mentioned above to study the occurrence of unstable modes during the shock wave computation. Readers are referred to the reference [14] for a more detailed description of the procedure.

Considering the numerical shock structure shown in Fig. 2. At time $t^{n}$, it is assumed that the instability happens and the intermediate states in cell $M$ are perturbed. The corresponding perturbation errors can be expressed as

$$
\rho_{M}^{n}=\rho_{M}^{*, n}+\delta \rho_{M}^{n}, \quad(\rho u)_{M}^{n}=(\rho u)_{M}^{*, n}+\delta(\rho u)_{M}^{n}, \quad p_{M}^{n}=p_{M}^{*, n}+\delta p_{M}^{n},
$$

where $\delta(\cdot)$ denote the perturbation errors which represent small discrepancies from the stable steady states. $(\cdot)^{*}$ denote the stable steady solutions that are assumed to locate at 
a Hugoniot curve. They have the following relationship with states in cell $L$ and cell $R$,

$$
\rho_{M}^{*, n}=\left(1-\alpha_{\rho}\right) \rho_{L}^{*, n}+\alpha_{\rho} \rho_{R}^{*, n}, \quad u_{M}^{*, n}=\left(1-\alpha_{u}\right) u_{L}^{*, n}+\alpha_{u} u_{R}^{*, n}, \quad p_{M}^{*, n}=\left(1-\alpha_{p}\right) p_{L}^{*, n}+\alpha_{p} p_{R}^{*, n},
$$

where the coefficients $\alpha_{\rho}, \alpha_{u}$ and $\alpha_{p}$ are defined in Eq. (3.4).

To explore how the perturbation errors generated in cell $M$ influence the mass flux perturbation in cell $R$, we need to consider the following conservative scheme

$$
(\rho u)_{R}^{n+1}=(\rho u)_{R}^{n}-\frac{\Delta t}{\Delta x}\left[\left(\rho u^{2}+p\right)_{R, R^{\prime}}^{n}-\left(\rho u^{2}+p\right)_{M, R}^{n}\right] .
$$

The subscript $R, R^{\prime}$ denotes the interface between the cell $R$ and the cell $R^{\prime}$, the subscript $M, R$ denotes the interface between the cell $M$ and the cell $R$. For the flux functions defined in Eqs. (2.7) and (2.10), the numerical fluxes at the interfaces in Eq. (3.8) can be written as

$$
\begin{aligned}
\left(\rho u^{2}+p\right)_{R, R^{\prime}}^{n}= & \left(\rho u^{2}+p\right)_{R^{\prime}}^{n} \\
\left(\rho u^{2}+p\right)_{M, R}^{n}= & \frac{S_{R}^{+}}{S_{R}^{+}-S_{M}^{-}}\left(\rho u^{2}+p\right)_{M}^{n}-\frac{S_{M}^{-}}{S_{R}^{+}-S_{M}^{-}}\left(\rho u^{2}+p\right)_{R}^{n}+\frac{S_{M}^{-} S_{R}^{+}}{S_{R}^{+}-S_{M}^{-}}\left[(\rho u)_{R}^{n}-(\rho u)_{M}^{n}\right] \\
& \quad-\frac{S_{M}^{-} S_{R}^{+}}{S_{R}^{+}-S_{M}^{-}}\left[\left(\rho_{R}^{n}-\rho_{M}^{n}-f_{X} \frac{p_{R}^{n}-p_{M}^{n}}{\widehat{a}^{2}}\right) \frac{\widehat{u}}{1+|\widehat{M}|}\right]
\end{aligned}
$$

with

$$
f_{\mathrm{X}}= \begin{cases}1, & \mathrm{X}=\text { Roe, } \\ f_{\text {RoeM }}, & \mathrm{X}=\text { RoeM, }\end{cases}
$$

where the subscript $M$ denotes the cell $M$, the subscript $R$ denotes the cell $R$ that is at the right side of $M . \widehat{(\cdot)}$ are Roe averaged variables between states in cells $M$ and $R$. $X$ represents a Roe-type scheme defined in Eqs. (2.7) or (2.10). Inserting Eq. (3.9) into Eq. (3.8), after some calculations we will obtain that

$$
\delta(\rho u)_{R}^{n+1}-\delta(\rho u)_{R}^{n}=\theta_{\rho} \cdot \delta \rho_{M}^{n}+\theta_{u} \cdot \delta u_{M}^{n}+\theta_{p} \cdot \delta p_{M}^{n}
$$

with

$$
\begin{aligned}
\theta_{\rho} & =\frac{S_{R}^{+} v M_{0}}{\left(S_{R}^{+}-S_{M}^{-}\right)\left(1+M_{0}\right)}\left(\frac{\omega^{2}}{f^{2}}-\frac{S_{M}^{-} \omega}{f}+\frac{S_{M}^{-}}{f(1+|\widehat{M}|)} \frac{1+\omega \sqrt{\sigma}}{1+\sqrt{\sigma}}\right), \\
\theta_{u} & =\frac{S_{R}^{+} v M_{0}}{\left(S_{R}^{+}-S_{M}^{-}\right)\left(1+M_{0}\right)}\left(2 \sigma \omega-S_{M}^{-} \sigma f\right), \\
\theta_{p} & =\frac{S_{R}^{+} v M_{0}}{\left(S_{R}^{+}-S_{M}^{-}\right)\left(1+M_{0}\right)}\left(1-f_{\mathrm{X}} \frac{S_{M}^{-}}{\widehat{a}^{2} f(1+|\widehat{M}|)} \frac{1+\omega \sqrt{\sigma}}{1+\sqrt{\sigma}}\right),
\end{aligned}
$$


where the coefficients $\omega$ and $\sigma$ are defined as

$$
\begin{aligned}
& \omega=f-f \cdot \alpha_{u}+\alpha_{u}, \\
& \sigma=\frac{1-\alpha_{\rho}+f \cdot \alpha_{\rho}}{f} .
\end{aligned}
$$

In Eq. (3.12), $v$ denotes the Courant number and the coefficients $\theta_{\rho}, \theta_{u}$ and $\theta_{p}$ are functions of the freestream Mach number $M_{0}$ and the shock position parameter $\varepsilon, f_{X}$ is defined in Eq. (3.10).

In our previous work [14], we have demonstrated that the erroneous mass flux behind the shock (i.e., the cell $R$ ) leads to the inconsistency of mass flux across the normal shock (i.e., $\left.(\rho u)_{L} \neq(\rho u)_{R}\right)$, which is found to be the cause of the shock instability. It can be observed from Eq. (3.11) that the perturbation errors of density, velocity and pressure all contribute to the mass flux perturbation error. The reason why the RoeM solver is able to improve the robustness of the original Roe scheme in the vicinity of strong shocks can also be clarified in the context of the linear perturbation analysis. That is the function $f_{\text {RoeM }}$ defined in Eq. (2.12) will become a small value, which plays a role in reducing the coefficient of the pressure perturbation $\theta_{p}$ and then a reduced mass flux in cell $R$ will be obtained. This simple cure for the shock instability of Roe scheme has an advantage over other improved Roe-type schemes, because only the pressure term in numerical flux is modified which will not introduce additional numerical dissipation on contact and shear waves. Actually, pressure is usually constant for the stationary contact discontinuity or the region normal to the wall in the boundary layer. However, the function $f_{\text {RoeM }}$ may be not enough to stabilize strong shocks. Table 1 shows the numerical results of the Roe-type schemes for stationary normal shock tests. Compared with the original Roe approach, the RoeM solver produces a larger stability spectrum, but it still exhibits shock instability for certain shock positions. In Fig. 3, we also provide the computed results from the Roe-type schemes. It shows that the normal shocks computed by the Roe and RoeM schemes are severely distorted. For the Roe scheme, the shock cannot even be fixed to its original position. The RoeM scheme is able to preserve the shock position but the profile is severely distorted. In the next section, we will propose a new modification to improve the robustness of the Roe approach, the main technique is to properly control the pressure perturbation by a new control function.

\subsubsection{An improved Roe-type scheme: RoeM+}

In the above section, we have demonstrated that the robustness of the Roe scheme for strong shocks can be improved by properly controlling the pressure perturbation as the RoeM does. Here, we propose a new improved Roe-type scheme which can be considered to be an enhancement of the RoeM solver. Thus, we denote the proposed method as RoeM+. The numerical flux function of the RoeM+ can be written as follows

$$
\boldsymbol{\Phi}_{\text {RoeM }+}=\frac{S_{R}^{+} \mathbf{H}_{n}\left(\mathbf{U}_{L}\right)-S_{L}^{-} \mathbf{H}_{n}\left(\mathbf{U}_{R}\right)}{S_{R}^{+}-S_{L}^{-}}+\frac{S_{R}^{+} S_{L}^{-}}{S_{R}^{+}-S_{L}^{-}} \Delta \mathbf{U}-\frac{S_{R}^{+} S_{L}^{-}}{S_{R}^{+}-S_{L}^{-}} \frac{1}{1+|\widehat{M}|} \mathbf{B} \Delta \mathbf{U},
$$


with

$$
\Delta \mathbf{U}=\Delta\left(\begin{array}{c}
\rho \\
\rho u \\
\rho v \\
E
\end{array}\right), \quad \mathbf{B} \Delta \mathbf{U}=\left(\Delta \rho-f_{\mathrm{RoeM}}+\frac{\Delta p}{\widehat{a}^{2}}\right)\left(\begin{array}{c}
1 \\
\widehat{u} \\
\widehat{v} \\
\frac{1}{2}\left|\widehat{q}_{n}\right|^{2}
\end{array}\right)+\widehat{\rho}\left(\begin{array}{c}
0 \\
\Delta u-\Delta \widehat{q}_{n} n_{x} \\
\Delta v-\Delta \widehat{q}_{n} n_{y} \\
\widehat{u} \Delta u+\widehat{v} \Delta v-\widehat{q}_{n} \Delta q_{n}
\end{array}\right),
$$

where the function $f_{\mathrm{RoeM}+}$ is used to control the pressure perturbation and it is defined as

$$
f_{\text {RoeM }+}=\min \left(p_{i, j+1 / 2}, p_{i-1 / 2, j}, p_{i+1 / 2, j}, p_{i-1 / 2, j+1}, p_{i+1 / 2, j+1}\right)^{3},
$$

with $p_{i, j+1 / 2}$ defined in Eq. (2.14). Furthermore, the numerical flux function defined in Eq. (3.14) and Eq. (3.15) can be modified to preserve total enthalpy in inviscid steady flow. This can be implemented by modifying the state vector and the dissipative terms in Eq. (3.15) as

$$
\Delta \mathbf{U}=\Delta\left(\begin{array}{c}
\rho \\
\rho u \\
\rho v \\
\rho H
\end{array}\right), \quad \mathbf{B} \Delta \mathbf{U}=\left(\Delta \rho-f_{\mathrm{Roe}}+\frac{\Delta p}{\widehat{a}^{2}}\right)\left(\begin{array}{c}
1 \\
\widehat{u} \\
\widehat{v} \\
\widehat{H}
\end{array}\right)+\widehat{\rho}\left(\begin{array}{c}
0 \\
\Delta u-\Delta \widehat{q}_{n} n_{x} \\
\Delta v-\Delta \widehat{q}_{n} n_{y} \\
\Delta H
\end{array}\right) .
$$

As argued by authors in $[28,60]$, the conservation of total enthalpy is preferred to predict hypersonic heating. Thus, we use (3.17) to evaluate the numerical flux $\boldsymbol{\Phi}_{\text {RoeM }+}$ in the current study. Compared with the function $f_{\text {RoeM }}$ defined in (2.12), the $f_{\text {RoeM }+}$ further reduces the perturbation coefficient of pressure $\theta_{p}$ and thus the perturbed mass flux will be suppressed more efficiently. The coefficient of pressure perturbation $\theta_{p}$ for the RoeM+ scheme can be obtained from Eq. (3.12) by replacing the $f_{\mathrm{X}}$ with $f_{\mathrm{RoeM}+}$. In Fig. 4, we give the coefficients of pressure perturbation $\theta_{p}$ of the Roe-type schemes as function of upstream Mach number $M_{0}$. As shown, the RoeM scheme produces smaller $\theta_{p}$ than the original Roe solver, but the coefficients exceed the stability threshold that is obtained from the matrix stability analysis in the next section. Contrasting to the Roe and RoeM method, the RoeM+ approach produces small enough $\theta_{p}$, they all lie inside the stable region. The numerical results in Table 1 show that the RoeM+ method is stable for the steady normal shock problem. In Fig. 3, it can be obtained that the RoeM+ solver is able to capture the shock without suffering any shock instability. In the following section, we will have a further study of the stability of these Roe-type schemes and demonstrate the validity of the improved method.

\subsection{A matrix stability analysis}

We have argued in the above section that the robustness of the Roe scheme can be improved by controlling the erroneous pressure perturbations that are transported downstream. To further justify this modification, we use a matrix-based stability analysis to 


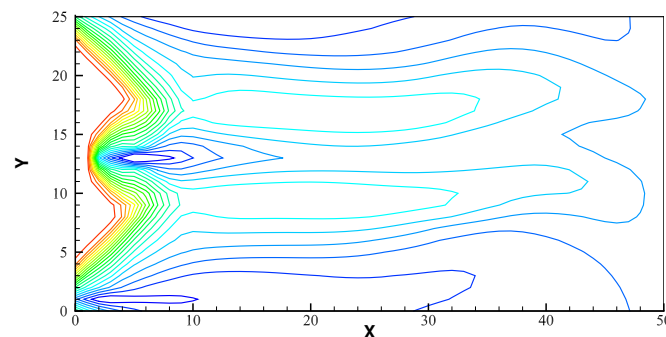

(a) Roe

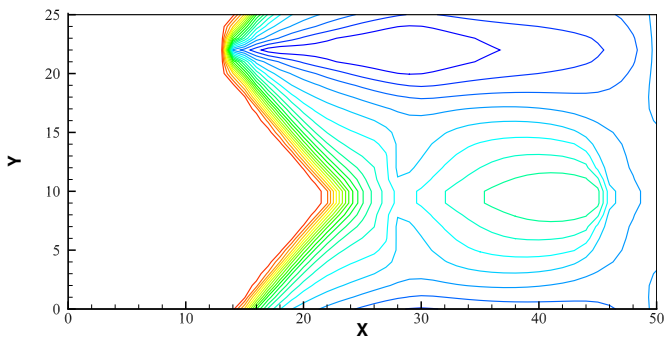

(b) RoeM

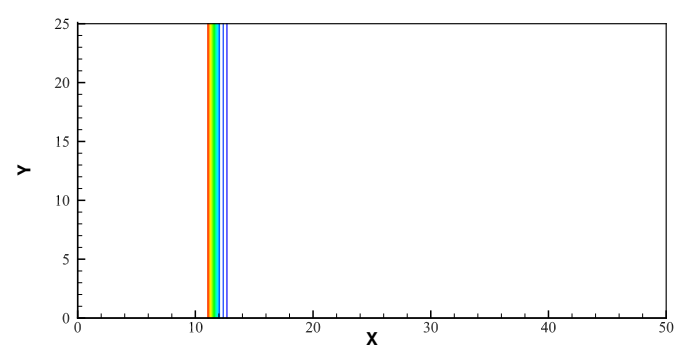

(c) RoeM+

Figure 3: Mach number contours for steady normal shock problem (grid with $50 \times 25$ cells, upstream Mach number $M_{0}=6.0$, shock position $\epsilon=0.3$ ).

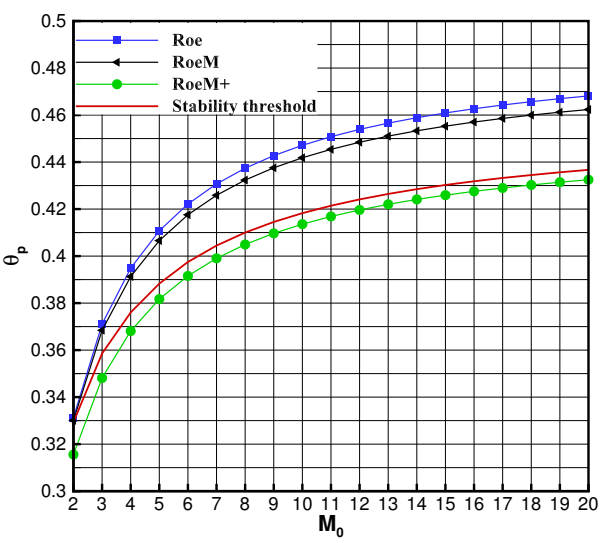

Figure 4: Coefficients of pressure perturbation vs. Mach number for different Roe-type schemes.

study the unstable behaviors of the Roe-type methods during the shock wave computation. To facilitate a further discussion, we briefly review the matrix-based method recommended in [30], and see more details in references [30,31].

Suppose the flow field is computed on the domain defined in Section 3.1, the numer- 
Table 1: Results of the steady normal shock problem for Roe-type flux functions at $M_{0}=6.0$ (S: stable and converged, U: unstable).

\begin{tabular}{||c|cccccccccc||}
\hline Roe-type schemes & $\varepsilon=0.0$ & 0.1 & 0.2 & 0.3 & 0.4 & 0.5 & 0.6 & 0.7 & 0.8 & 0.9 \\
\hline Roe & $\mathrm{U}$ & $\mathrm{U}$ & $\mathrm{U}$ & $\mathrm{U}$ & $\mathrm{U}$ & $\mathrm{U}$ & $\mathrm{U}$ & $\mathrm{S}$ & $\mathrm{S}$ & $\mathrm{S}$ \\
RoeM & $\mathrm{U}$ & $\mathrm{U}$ & $\mathrm{U}$ & $\mathrm{U}$ & $\mathrm{U}$ & $\mathrm{S}$ & $\mathrm{S}$ & $\mathrm{S}$ & $\mathrm{S}$ & $\mathrm{S}$ \\
RoeM+ & $\mathrm{S}$ & $\mathrm{S}$ & $\mathrm{S}$ & $\mathrm{S}$ & $\mathrm{S}$ & $\mathrm{S}$ & $\mathrm{S}$ & $\mathrm{S}$ & $\mathrm{S}$ & $\mathrm{S}$ \\
\hline
\end{tabular}

ical setup is also used. For the stability analysis of a steady field, we assume that the domain is submitted to small numerical random errors as

$$
\mathbf{U}_{i}=\mathbf{U}_{i}^{0}+\delta \mathbf{U}_{i},
$$

where $\mathbf{U}_{i}^{0}$ is the steady mean value and $\delta \mathbf{U}_{i}$ represents the small numerical random perturbation. The flux function can be linearized around the steady mean value as follows

$$
\boldsymbol{\Phi}_{i j}\left(\mathbf{U}_{i}, \mathbf{U}_{j}\right)=\boldsymbol{\Phi}_{i j}\left(\mathbf{U}_{i}^{0}, \mathbf{U}_{j}^{0}\right)+\frac{\partial \boldsymbol{\Phi}_{i j}}{\partial \mathbf{U}_{i}} \delta \mathbf{U}_{i}+\frac{\partial \boldsymbol{\Phi}_{i j}}{\partial \mathbf{U}_{j}} \delta \mathbf{U}_{j} .
$$

A linear error evolution model can be obtained by combing Eqs. (2.4), (3.18) and (3.19), it is written by

$$
\frac{d\left(\delta \mathbf{U}_{i}\right)}{d t}=-\frac{1}{\left|\Omega_{i}\right|} \sum_{\Gamma_{i j} \subset \partial \Omega_{i}}\left|\Gamma_{i j}\right|\left(\frac{\partial \boldsymbol{\Phi}_{i j}}{\partial \mathbf{U}_{i}} \delta \mathbf{U}_{i}+\frac{\partial \boldsymbol{\Phi}_{i j}}{\partial \mathbf{U}_{j}} \delta \mathbf{U}_{j}\right) .
$$

The first term on the right hand side of (3.20) contains the influence of the error in the cell $i$ itself, and the second term contains the influence of the errors in the neighbour cells $j$. For the computational domain of the stationary normal shock problem, there is $j=4$. Eq. (3.20) holds for all cells in the computational domain and so we finally get the error evolution of all $i$ cells in the domain

$$
\frac{d}{d t}\left(\begin{array}{c}
\delta \mathbf{U}_{1} \\
\vdots \\
\delta \mathbf{U}_{i}
\end{array}\right)=S\left(\begin{array}{c}
\delta \mathbf{U}_{1} \\
\vdots \\
\delta \mathbf{U}_{i}
\end{array}\right)
$$

where $S$ is the stability matrix. When considering only the evolution of initial errors, the solution of the linear time invariant system (3.21) is

$$
\left(\begin{array}{c}
\delta \mathbf{U}_{1} \\
\vdots \\
\delta \mathbf{U}_{i}
\end{array}\right)(t)=\exp ^{S t}\left(\begin{array}{c}
\delta \mathbf{U}_{1} \\
\vdots \\
\delta \mathbf{U}_{i}
\end{array}\right)_{t=0}
$$

and remains bounded if the maximum of the real part of the eigenvalues of $S$ is nonpositive

$$
\max (\operatorname{Re}(\lambda(S))) \leq 0
$$




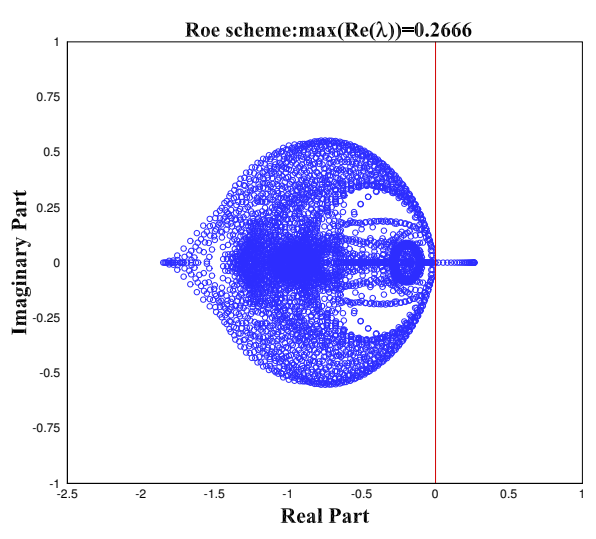

(a) Roe

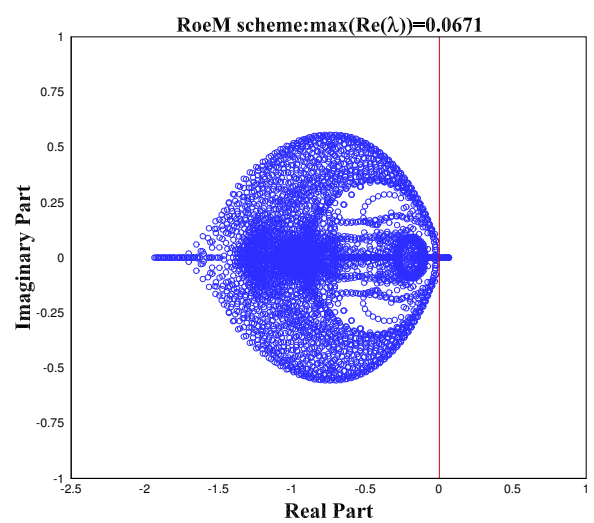

(b) RoeM

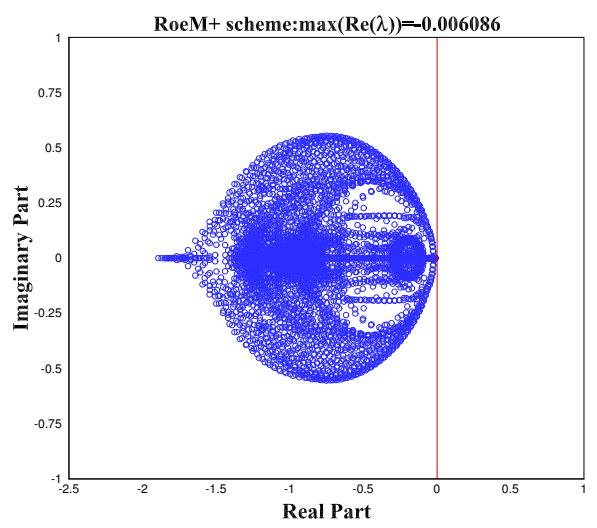

(c) RoeM+

Figure 5: Distribution of the eigenvalues of stability matrix in the complex plane for Roe-type schemes (grid with $50 \times 25$ cells, upstream Mach number $M_{0}=6.0$, shock position $\varepsilon=0.3$ ).

The stability of the Roe-type schemes in the current study has been analyzed. In Fig. 5, we present the distribution of the eigenvalues of the stability matrix in the complex plane for different schemes. It can be observed that the original Roe scheme has a maximum eigenvalue of 0.2666 , which is responsible for its shock instability. For the RoeM approach, the maximum eigenvalue is reduced to 0.0671 , which corresponds to its improved robustness for strong shocks, but it is still unstable. With our new modification, the maximum eigenvalue of the proposed scheme RoeM+ is -0.006086 , demonstrating its stability.

In Fig. 6, we present the maximal real parts of the eigenvalues of the Roe-type schemes as functions of the freestream Mach number. It can be observed that the Roe and RoeM schemes are always unstable when the upstream Mach number is greater than about 2 . 


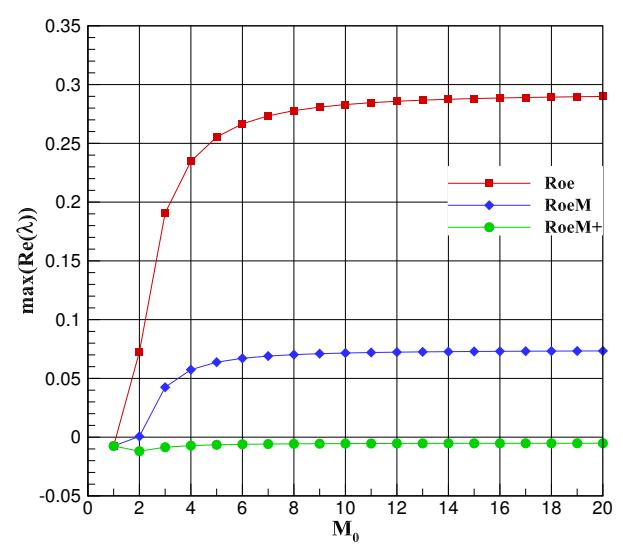

Figure 6: Maximal real part of eigenvalues of stability matrix vs. Mach number.

However, the RoeM+ scheme is always stable for all Mach numbers. These results show that the pressure perturbation-control technique is efficient to suppress the numerical shock instability and this further justifies the above linear perturbation analysis.

\section{Low Mach number extension}

Godunov-type schemes developed for compressible flows usually encounter the accuracy problem in the low Mach number limit. It has been well demonstrated in [32] that the Roe scheme fails to handle low Mach number flows. This problem can be cured by applying an all Mach correction to the numerical flux. Here, we extend the RoeM+ scheme defined in Eqs. (3.14)-(3.16) and Eq. (2.16) to simulate low Mach number flows. The essential ingredient of the extension is the all Mach correction technique proposed by Dellacherie et al. [36].

We consider the following Godunov-type scheme that solves the compressible Euler equations

$$
\frac{d \mathbf{U}_{i}}{d t}+\frac{1}{\left|\Omega_{i}\right|} \sum_{\Gamma_{i j} \subset \partial \Omega_{i}}\left|\Gamma_{i j}\right| \Phi_{i j}^{\mathrm{AM}, \mathrm{X}}=0,
$$

where $X$ denotes a Godunov-type scheme. According to Dellacherie et al. [36], the all Mach flux function $\boldsymbol{\Phi}_{i j}^{\mathrm{AM}, \mathrm{X}}$ can be expressed as

$$
\boldsymbol{\Phi}_{i j}^{\mathrm{AM}, \mathrm{X}}=\boldsymbol{\Phi}_{i j}^{\mathrm{X}}+\left(1-\theta_{i j}\right) \frac{\rho_{i j} a_{i j}}{2}\left(\begin{array}{c}
0 \\
\Delta q_{n} n_{x} \\
\Delta q_{n} n_{y} \\
0
\end{array}\right),
$$


where $\boldsymbol{\Phi}_{i j}^{X}$ represents the unmodified flux given by the X scheme. The $\theta_{i j}$ is the weight function that is defined as

$$
\theta_{i j}=\theta\left(M_{i j}\right) \quad \text { with } \theta(M)=\min (M, 1) .
$$

In Eqs. (4.2) and (4.3), $M_{i j}, \rho_{i j}$ and $a_{i j}$ are the calculated Mach number, density and sound speed at the interface and $\Delta q_{n}$ denotes the normal velocity difference, i.e., $\Delta q_{n}=\left(q_{n}\right)_{j}-$ $\left(q_{n}\right)_{i}$. Following $[37,38]$, the local Mach number is defined as

$$
M_{i j}=\max \left(\sqrt{u_{i}^{2}+v_{i}^{2}} / a_{i}, \sqrt{u_{j}^{2}+v_{j}^{2}} / a_{j}\right) .
$$

Here, we use the all Mach correction defined in Eqs. (4.2)-(4.4) to cure the accuracy problem of RoeM+ scheme at low Mach numbers and add some improvements. The resulting all Mach Roe-type scheme denoted as AM-RoeM+ can be written by

$$
\boldsymbol{\Phi}_{i j}^{\mathrm{AM}-\mathrm{RoeM}+}=\boldsymbol{\Phi}_{i j}^{\mathrm{RoeM}+}+f_{\mathrm{RoeM}+}\left(1-\theta_{i j}\right) \frac{\rho_{i j} a_{i j}}{2}\left(\begin{array}{c}
0 \\
\Delta q_{n} n_{x} \\
\Delta q_{n} n_{y} \\
0
\end{array}\right),
$$

where $\theta_{i j}$ is defined in Eq. (4.3) and Eq. (4.4), $f_{\text {RoeM }+}$ is defined in Eq. (3.16). Here, $(\cdot)_{i j}$ are calculated by Roe averaged method. As argued by Oßwald et al. [42], the modified term for low Mach flow may trigger small disturbances in the vicinity of shocks. To avoid this anomaly, the function $f_{\mathrm{RoeM}+}$ is added to the correction term to turn off the low Mach correction in the vicinity of strong shocks. Note that the resulting all Mach Roe-type scheme does not involve any cut-off reference Mach number and user-defined parameters.

\section{Numerical results}

In this section, we present numerical results of the proposed Roe-type scheme for different kinds of test cases to demonstrate the capabilities. The first two test problems focus on the entropy satisfaction and the positivity preservation properties of the numerical methods. The following two test cases are selected to demonstrate the robustness of the numerical schemes against strong shocks. In the next two tests, the accuracy of the all Mach Roe-type scheme in the low Mach number regime is demonstrated. In the last two cases, we present results for viscous flow problems, a focus is on accuracy for resolving shear layers.

\subsection{A modified Sod's shock tube problem}

We first consider a modified version of the popular Sod's shock tube problem [52]. The solution consists of a right shock wave, a right traveling contact wave and a left sonic 


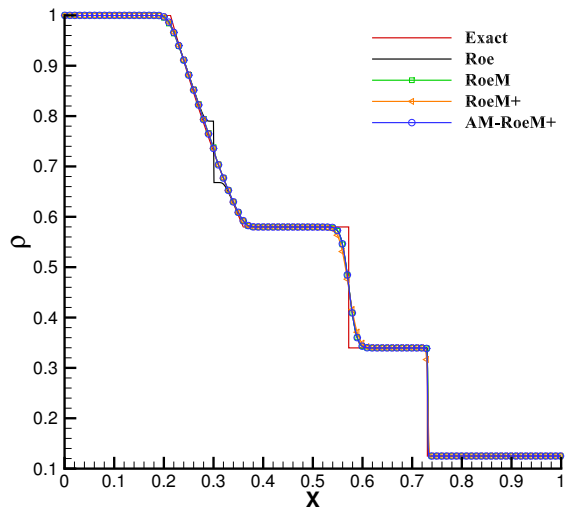

(a)

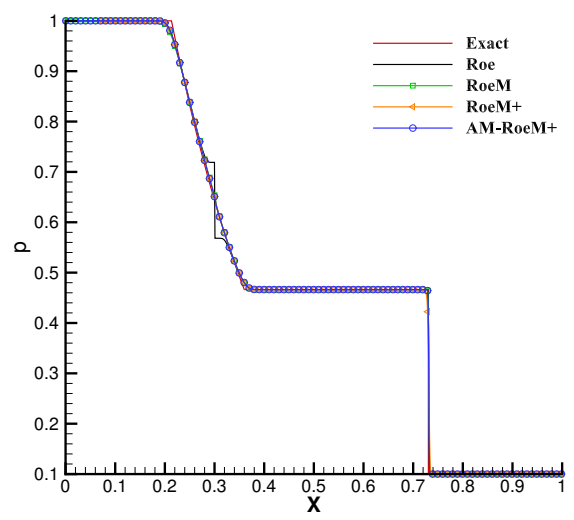

(c)

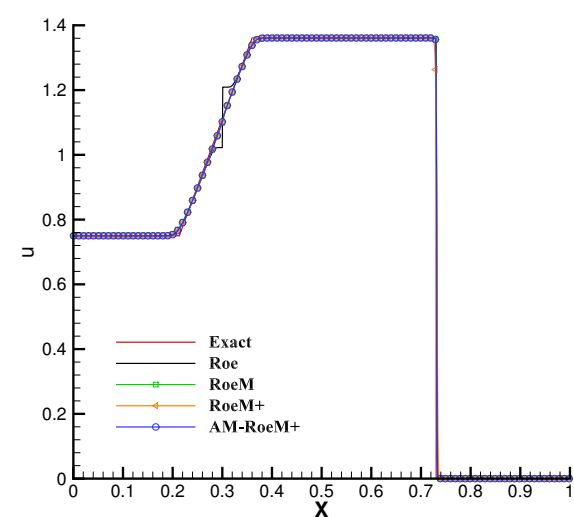

(b)

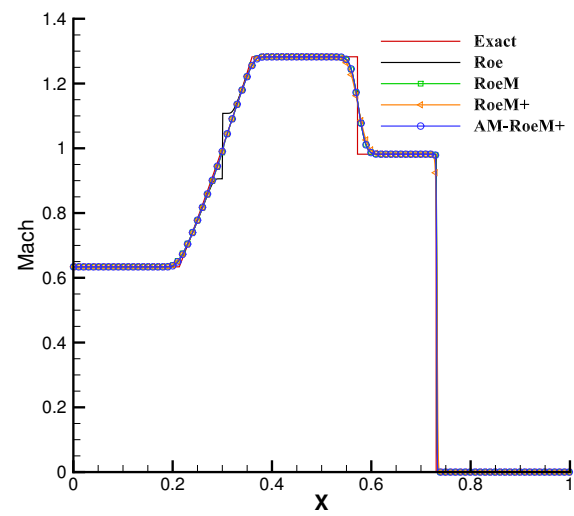

(d)

Figure 7: Modified Sod's shock tube problem at time $t=0.2 \mathrm{~s}$, (a) density profiles, (b) velocity profiles, (c) pressure profiles and (d) Mach number profiles.

rarefaction wave. This test is very useful in assessing the entropy satisfaction property of numerical methods. The initial conditions are

$$
\left(\rho_{L}, u_{L}, p_{L}\right)=(1.0,0.75,1.0) \quad \text { and } \quad\left(\rho_{R}, u_{R}, p_{R}\right)=(0.125,0.0,0.1)
$$

The discontinuity is set at the position $x=0.3$. The computational domain is equal to $[0,1]$, which contains $N=1000$ regular cells. The Courant number coefficient is $C F L=0.9$, non-reflecting boundary conditions are used at the boundaries. In Fig. 7, we compare all the Roe-type schemes in the current study to the exact solution at time $t=0.2 \mathrm{~s}$.

The solutions of these Roe-type schemes are presented in Fig. 7. One can see that the Roe scheme produces a nonphysical expansion shock as anticipated, the RoeM, RoeM+ 
and AM-RoeM+ schemes show a correct agreement with the exact solution without admitting any discontinuity in the left rarefaction wave. The resulting Mach number varies from 0 to 1.28, so both low Mach and order one Mach values exist in the whole computational domain. The all Mach Roe-type scheme, i.e., AM-RoeM+, is stable and produces right numerical solutions.

\subsection{Double rarefaction problem}

In our second test case, we use the double rarefaction problem [10] to assess the performance of numerical methods for low density flows. This test consists of two rarefaction waves and a trivial contact wave of zero speed. The region between the two nonlinear waves is close to vacuum. The initial conditions are

$$
\left(\rho_{L}, u_{L}, p_{L}\right)=(1.0,-2.0,0.4) \quad \text { and } \quad\left(\rho_{R}, u_{R}, p_{R}\right)=(1.0,2.0,0.4) .
$$

The spatial domain is the interval $[0,1]$, which is discretized with $N=1000$ computing cells. The Courant number coefficient is set as $\mathrm{CFL}=0.9$, boundary conditions are transmissive.

In Fig. 8, we compare the Roe-type schemes to the exact solution at time $t=0.15 \mathrm{~s}$. The resulting Mach number varies from 0 to 2.7, so we have both low Mach and order one Mach values. The original Roe solver fails on this test, so its results are not shown. The AM-RoeM+ approach produces almost the same solutions as the RoeM and RoeM+ schemes do. All these schemes show a correct agreement with the exact solution and they preserve the positivity of the solution.

\subsection{Hypersonic inviscid flow past a cylinder}

Hypersonic inviscid flow past a cylinder is a typical problem to assess the robustness of Godunov-type schemes against shock anomalies such as the carbuncle phenomenon. Here, we use the numerical setup of this problem from Ohwada et al. [53]. It has been shown in [53] that this simple setting poses a challenge to many Godunov-type schemes, including the AUSM+ scheme [54], the AUSM+-up scheme [55], the SLAU scheme [26], the HLLC scheme [56], etc. The setting of the problem is as follow. A cylinder with a radius of the reference length is located in a uniform gas where the upstream Mach number is set as 8 . The axis of the cylinder is at the origin $(x, y)=(0,0)$. The following mesh system is used to define and discrete the computational domain,

$$
\begin{aligned}
& \bar{x}=-(3.8-2.8 \xi) \cos \eta, \\
& \bar{y}=(3.8-2.8 \xi) \sin \eta, \\
& \frac{1}{2} \leq \xi \leq 1, \quad-\frac{2 \pi}{5} \leq \eta \leq \frac{2 \pi}{5},
\end{aligned}
$$

where the spatial coordinates are normalized by the radius of the cylinder and the intervals for $\xi$ and $\eta$ are uniformly divided into 120 and 320 sections. The computational 


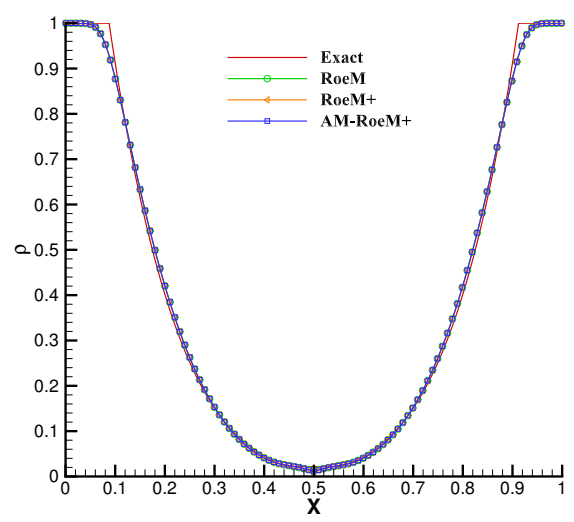

(a)

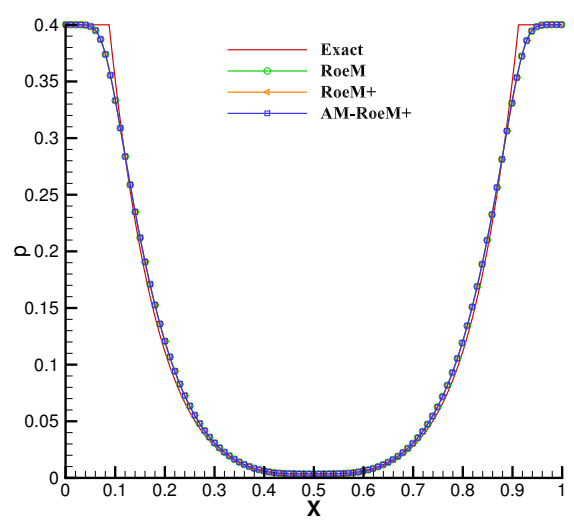

(c)

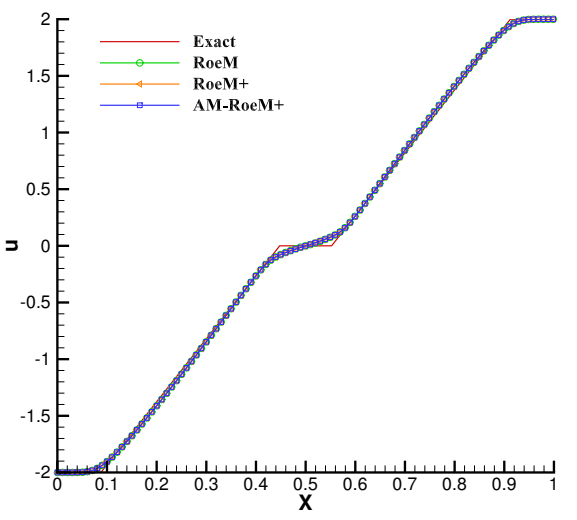

(b)

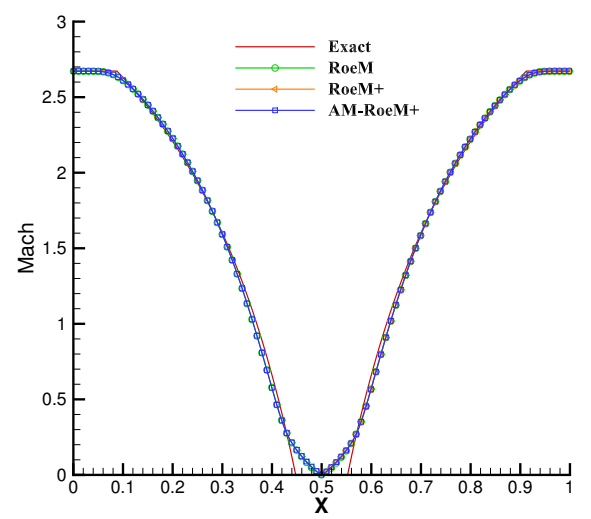

(d)

Figure 8: A vacuum test problem at time $t=0.15 \mathrm{~s}$, (a) density profiles, (b) velocity profiles, (c) pressure profiles and (d) Mach number profiles.

domain has been initialized with values $\rho=1.4, p=1, u=8$ and $v=0$. At the wall, the slip condition is used and the other two are taken as outflow. The simulations are conducted in first-order accurate schemes with $C F L=0.5$.

In Fig. 9, the density contours by three Roe-type schemes and the HLLE scheme are illustrated, where 20 contour levels varying from 2.0 to 8.0 are used. The computational result from the HLLE scheme with the wave speeds estimate defined in (2.16) is taken as a reference solution due to its reliability and robustness in capturing strong shocks. The original Roe scheme fails this test case thus its result is not shown. As shown in Fig. 9, the RoeM scheme exhibits the carbuncle phenomenon, a spurious bump is clearly demonstrated near the stagnation streamline. Both the RoeM+ and the AM-RoeM+ schemes 


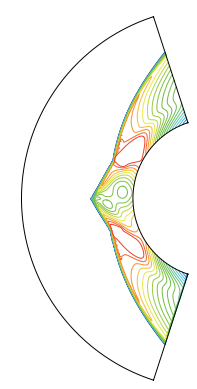

(a) RoeM

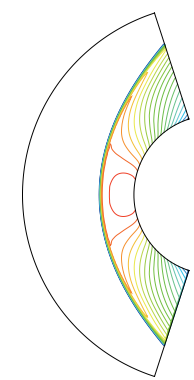

(c) $\mathrm{AM}-\mathrm{RoeM}+$

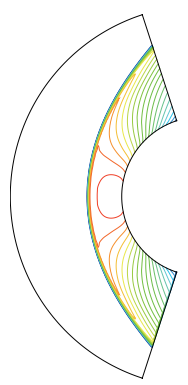

(b) RoeM+

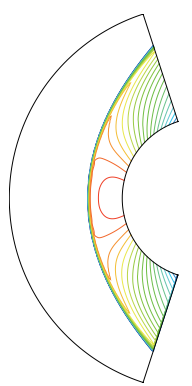

(d) HLLE

Figure 9: The density contours for hypersonic flow over a cylinder, (a) RoeM, (b) RoeM+, (c) AM-RoeM+, (d) HLLE.

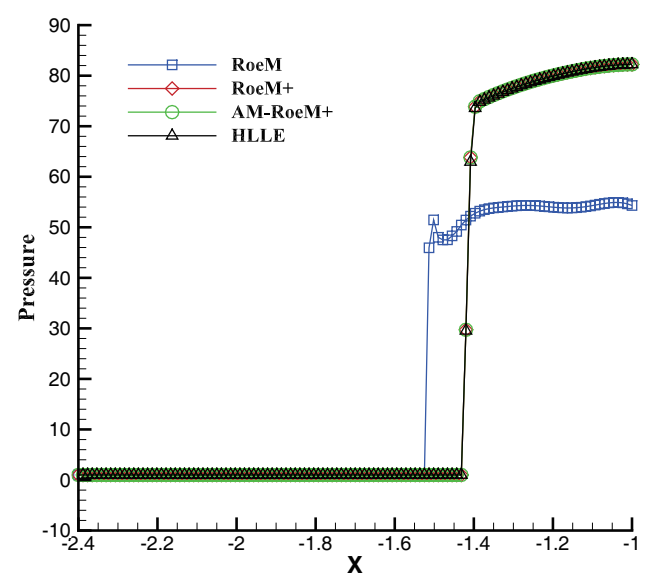

Figure 10: The pressure profiles along the stagnation line for different schemes.

produce clean shock profiles and their post shock regions are free from any shock anomalies. They show a fairly high level of robustness as the HLLE scheme does. In Fig. 10, the pressure profiles along the stagnation line for different schemes are plotted. As shown, 


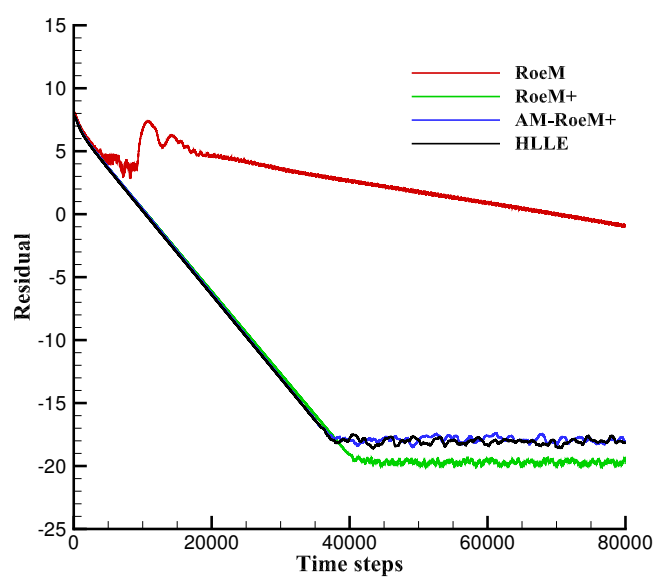

Figure 11: Convergence histories for different schemes with the inflow Mach number 8.

the RoeM solver is not able to provide a correct pressure profile, the RoeM+ scheme and the AM-RoeM+ scheme produce almost identical results to that of the HLLE scheme. A comparison of residual convergence histories from different schemes is given in Fig. 11. The improved Roe-type schemes, i.e., RoeM+ and AM-RoeM+ both display well-behaved convergence histories. In particular, the AM-RoeM+ scheme is able to produce the almost identical convergence rate to that of the HLLE scheme.

\subsection{Double Mach reflection problem}

Many numerical schemes are claimed to produce kinked Mach stems in the double Mach reflection problem. It is first studied by Woodward and Colella [57] and followed by many other scholars. The problem describes a planar shock wave propagating in inviscid fluid which is reflected by a $30^{\circ}$ ramp. The computational domain is $[0,4] \times[0,1]$ which has been divided into 480 cells along the length and 120 cells along the width. The shock with a Mach number of 10 is initially set up to be inclined at an angle of 60 with the bottom reflecting wall. The domain in front of the shock is initialized with pre shock values given as $\rho=1.4, u=0, v=0, p=1$. The domain behind the shock is initialized to post shock values. The computations are performed by first-order numerical schemes and the third-order TVD Runge-Kutta time discretization [58] with $C F L=0.5$ up to $t=0.2 \mathrm{~s}$. The density contours computed by different schemes are shown in Fig. 12, where 30 contour levels varying from 2.0 to 20.0 are used. As shown, the Roe scheme produces a visible kinked Mach stem. The density contour computed by the RoeM scheme demonstrates an improved result, but the kinked Mach stem is still clearly visible. In contrast to the above two Roe-type schemes, the RoeM+ and the AM-RoeM+ schemes produce almost identical solutions, the kinked Mach stems shown in their density contours are barely noticeable. 


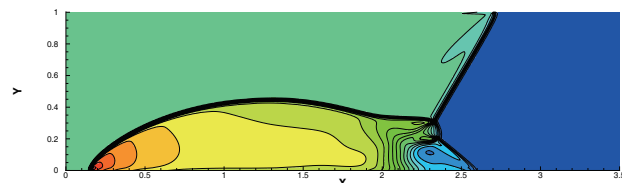

(a) Roe

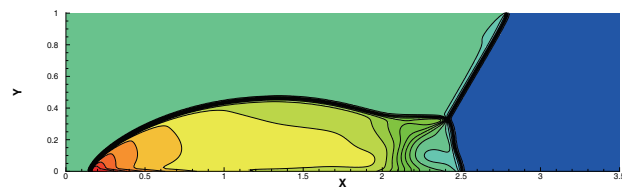

(c) RoeM+

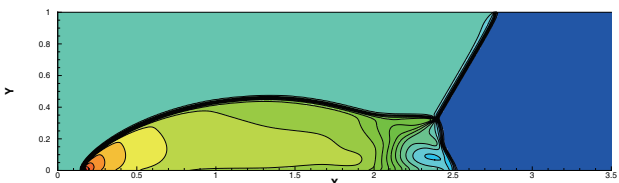

(b) RoeM

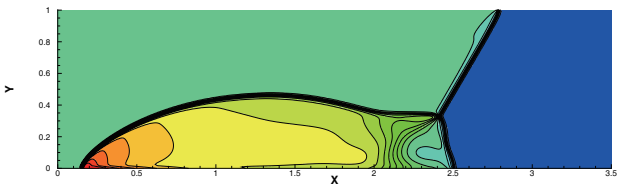

(d) $\mathrm{AM}-\mathrm{RoeM}+$

Figure 12: The density contours for double Mach reflection problem at $t=0.2 \mathrm{~s}$.

\subsection{Gresho vortex}

To exhibit the low-speed performance of the Roe-type schemes, we consider a rotating flow in form of the Gresho vortex [59]. Here, we follow the setup of this test case in [34] closely. The computational domain is $\Omega=[0,1] \times[0,1]$ with an initial background state given by

$$
\rho_{0}=1.0, \quad \mathbf{u}_{0}=\left(u_{0}, 0\right)^{T}, \quad p_{0}=1.0, \quad a_{0}=\sqrt{\gamma p_{0} / \rho_{0}}=\sqrt{\gamma}, \quad u_{0}=M_{0} a_{0},
$$

where $M_{0}$ is the global Mach number. At the initial time, a vortex of radius $R=0.4$ is centered at $\left(x_{0}, y_{0}\right)=(0.5,0.5)$. The initial condition is given as:

$$
u_{r}(r)=u_{0} \begin{cases}2 r / R, & \text { if } 0 \leq r<R / 2 \\ 2(1-r / R), & \text { if } R / 2 \leq r<R \\ 0, & \text { if } R \leq r\end{cases}
$$

and the initial pressure field is set as

$$
p(r)=p_{0}+u_{0}^{2} \begin{cases}2 r^{2} / R^{2}+2-\log 16, & \text { if } 0 \leq r<R / 2, \\ 2 r^{2} / R^{2}-8 \frac{r}{R}+4 \log \frac{r}{R}+6, & \text { if } R / 2 \leq r<R \\ 0, & \text { if } R \leq r\end{cases}
$$

where the radius $r$ is written by

$$
r=\sqrt{\left(x-x_{0}\right)^{2}+\left(y-y_{0}\right)^{2}} .
$$

Periodic boundary conditions are imposed on the left and right boundaries. At lower and upper boundaries, far-field boundary conditions are imposed by setting the initial 
conditions to the ghost cells. The simulations of the test case are carried out on a $100 \times 100$ Cartesian mesh for the Mach numbers $M_{0}=10^{-2}$. The forward Euler scheme is used for the temporal discretization. The CFL number is set as 0.9 .

We use a normalized pressure field defined as

$$
p_{N}(x, y)=\frac{p(x, y)-p_{\min }}{p_{\max }-p_{\min }}
$$

to characterize the order of pressure fluctuations in the incompressible limit. The Gresho vortex as set up here is a stationary solution to the incompressible Euler equations. Thus the normalized pressure field should not change with time. We therefore use this problem to check the quality of different Riemann solvers in the regime of low Mach numbers. In Fig. 13, the normalized pressure fields for Gresho vortex at $M_{0}=0.01$ after one period are shown. Due to the excessive numerical dissipation of the Roe-type schemes, the vortices are highly inaccurate and completely dissipated after one period. However, the improved solver AM-RoeM+ provides better accuracy than the Roe solver. The normalized pressure contour of the AM-RoeM+ scheme for $M_{0}=10^{-2}$ is almost identical to the initial state. The normalized pressure distributions along the horizontal centerline for Gresho vortex are extracted shown in Fig. 14. It is observed that the proposed method AM-RoeM+ is able to resolve the vortex with less numerical dissipation and achieve better accuracy compared to the other Roe-type schemes.

\subsection{Low Mach number flows around the NACA0012 airfoil}

In order to further examine the performance of numerical schemes in the regime of low Mach number flow, we present inviscid flows around NACA0012 airfoil at a sequence of low Mach numbers. An O-type mesh with 241 (circumferential) $\times 121$ (normal) cell number is used for all the computations. The outer boundary is 19 chord lengths away from the airfoil wall. The slip boundary conditions are used on the wall. The simulations are conducted at a 0 angle of attack, where four Mach numbers: $M_{0}=0.1,0.01,0.001,0.0001$ are set as inflow conditions. The computations are conducted for 50,000 time steps with a CFL number of 10 using the LU-SGS approach. At least five orders of density-residual reduction is achieved.

Fig. 15 shows the normalized pressure contours of the test case. It can be observed that the original Roe scheme, the RoeM scheme and the RoeM+ scheme all fail to simulate the low Mach number flows accurately, whereas the modified scheme AM-RoeM+ converges to a solution that approaches the incompressible flow. Fig. 16 shows the behaviour of the pressure fluctuations with the inflow Mach number. As we know, first order Riemann solvers for compressible flows usually support pressure fluctuations in space of order $M_{0}$, whereas the physical pressure fluctuation should scale as $M_{0}^{2}$. As shown in Fig. 16, the original Roe solver gives the incorrect scaling of the pressure fluctuations. With the improved Roe-type scheme AM-RoeM+, the pressure fluctuations exactly scale with $M_{0}^{2}$. 


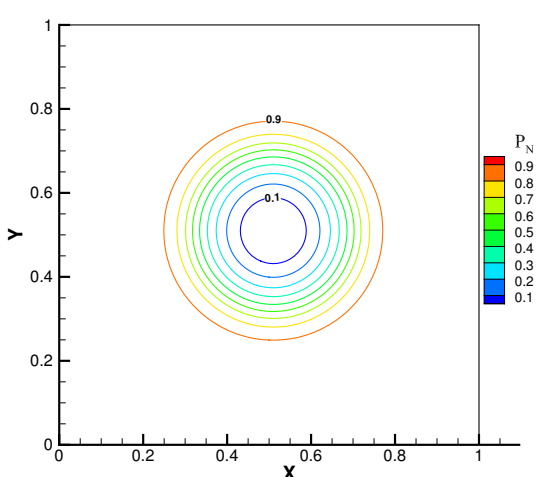

(a)

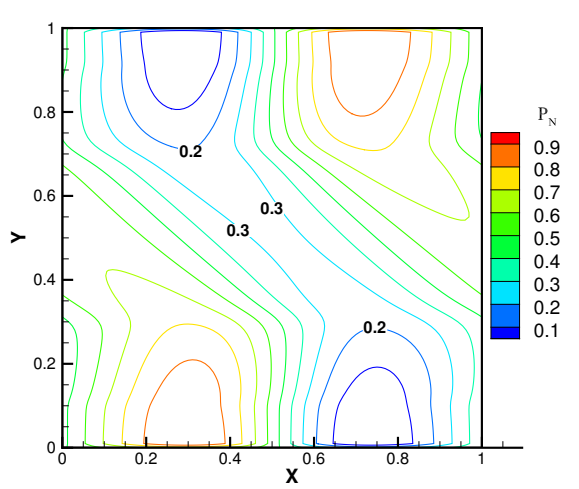

(c)

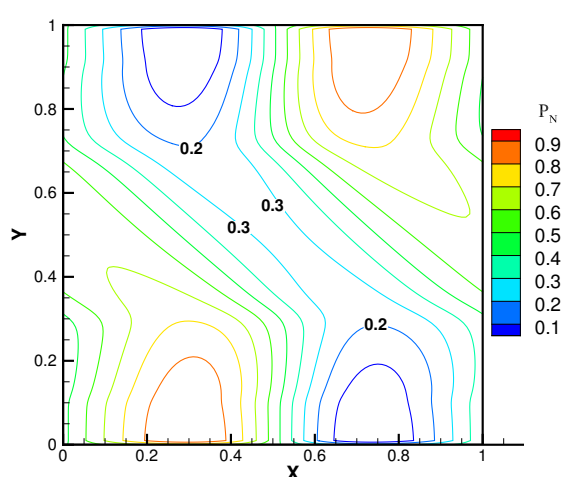

(b)

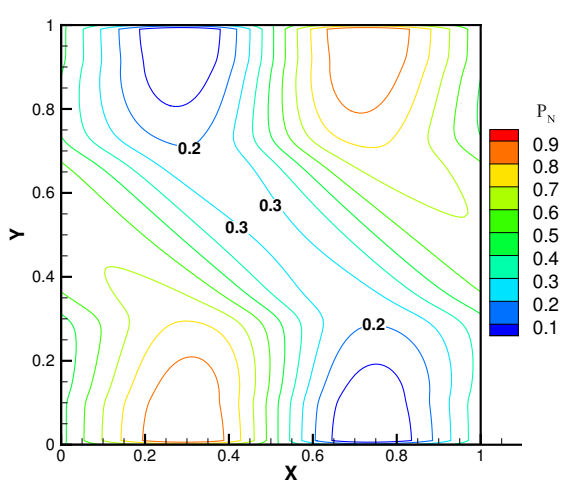

(d)

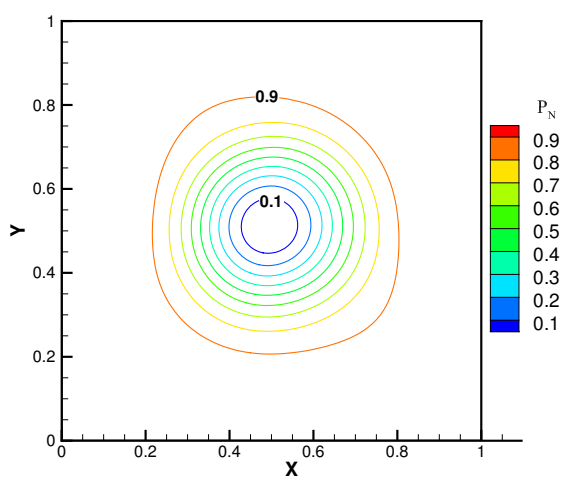

(e)

Figure 13: The normalized pressure isolines for Gresho vortex with the explicit first-order schemes for $M_{0}=0.01$ after one period: (a) Initial condition, (b) Roe, (c) RoeM, (d) RoeM+, (e) AM-RoeM+. 


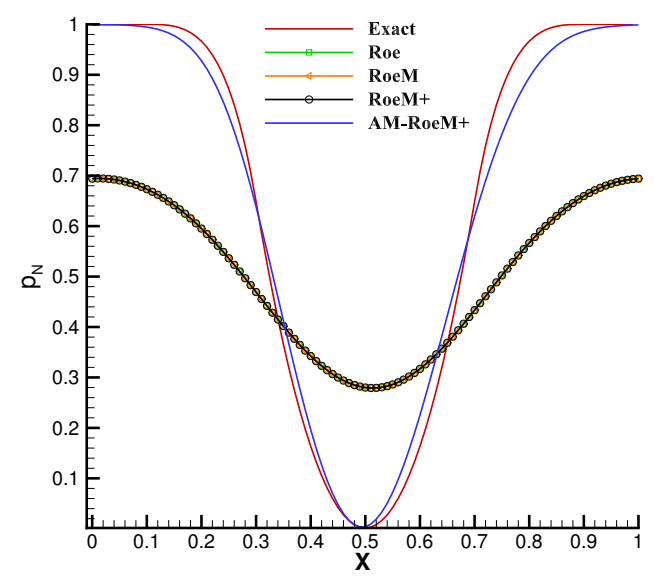

Figure 14: Normalized pressure distributions along the horizontal centerline for Gresho vortex with $M_{0}=0.01$ after one period.

\subsection{Laminar boundary layer}

To further demonstrate the ability of the proposed method to resolve shear layers accurately, a laminar boundary layer is tested over a flat plate with length $L=100$. The freestream Mach number is $M a=0.3$ and the Reynolds number is $R e=U_{\text {inf }} L / v=10^{5}, v$ is the viscous coefficient. This problem is simulated using a second-order Navier-Stokes code for a rectangular mesh with $120 \times 30$ non-uniform grid points. Fig. 17 presents the computational mesh. As shown, the non-slip adiabatic boundary condition is imposed at the plate and a symmetry condition is used at the bottom boundary before the flat plate. The non-reflecting boundary condition based on the Riemann invariants is adopted for other boundaries. The computations were conducted for 50,000 steps with $C F L=0.5$, and all the computations achieved at least three orders of magnitude reductions of the density residuals. The results of different schemes are compared in Fig. 18, as well as Blasius' analytical solution for a laminar boundary layer. As shown, the proposed scheme AMRoeM+ can successfully reproduce the analytical velocity profile just as the Roe scheme and the RoeM scheme do. Actually, the results of these three solvers are almost identical. The result of the HLLE scheme is also included in Fig. 18 for comparison. As expected, the HLLE scheme produces a very dissipative and inaccurate solution due to its dissipative property.

\subsection{Hypersonic viscous flow past a cylinder}

In the last test case, we will conduct numerical computations of a hypersonic viscous flow past a cylinder to examine the performance of the proposed AM-RoeM+ solver. The numerical setup is the same as that in references $[53,60]$. The freestream conditions are given as $M_{\text {inf }}=8.1, P_{\text {inf }}=370.7 p a, T_{\text {inf }}=63.73 \mathrm{~K}$ for the far field, and the Reynolds 


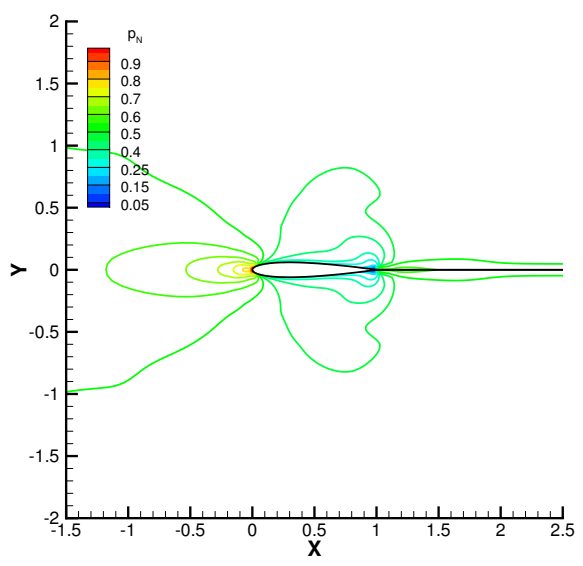

(a)

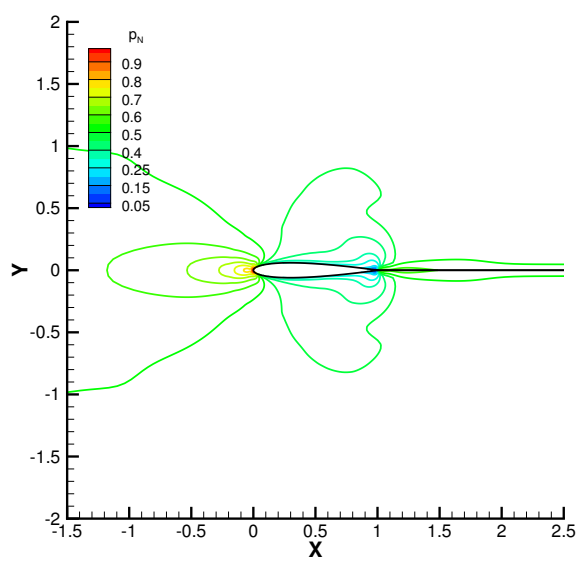

(c)

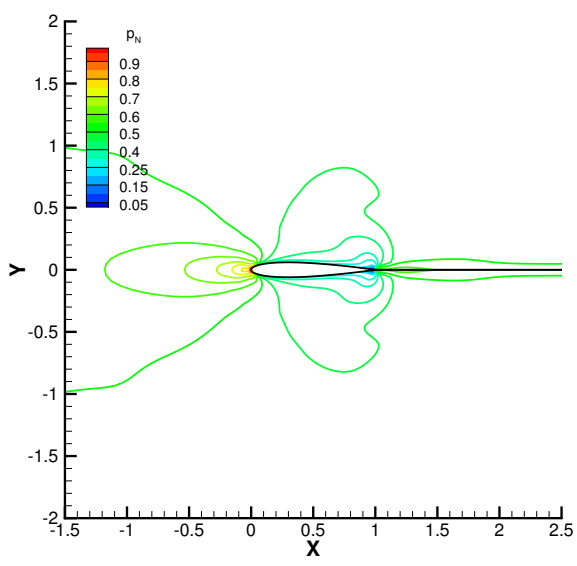

(b)

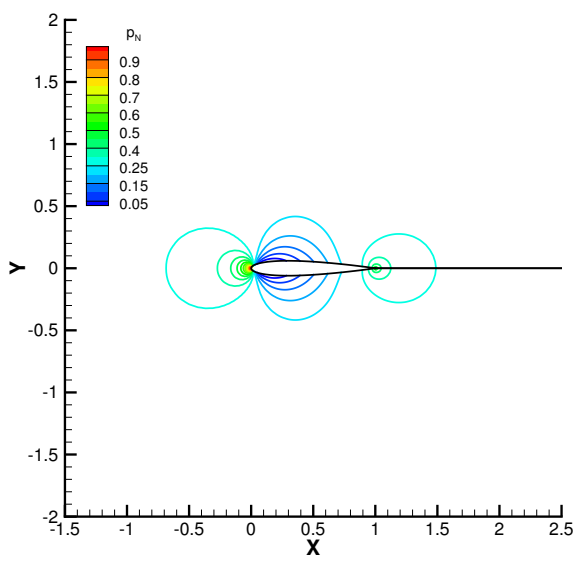

(d)

Figure 15: The normalized pressure isolines for inviscid flows around NACA0012 airfoil at Mach 0.001 by the first-order schemes: (a) Roe; (b) RoeM; (c) RoeM+; (d) AM-RoeM+.

number based on the radius $(r=20 \mathrm{~mm})$ of the cylinder and the far field flow parameters is $1.3 \times 10^{5}$. The non-slip and isothermal conditions with the wall temperature $T_{w}=300 \mathrm{~K}$ are imposed at the wall; the Prandtl number of the gas is taken as 0.72; the temperature dependence of the viscosity is taken into account by using the Sutherland formula. The computational mesh adopted here is the same as that in the inviscid case (see Section 5.3), but the grids are refined near the cylinder surface to resolve the boundary layer well. Following Ohwada et al. [53], the non-uniform grid for $\xi$ is introduced in the mesh defined in Eq. (5.1) as

$$
\xi=\frac{81-41 \exp (-s)}{80}, \quad 0 \leq s \leq \ln (41)
$$




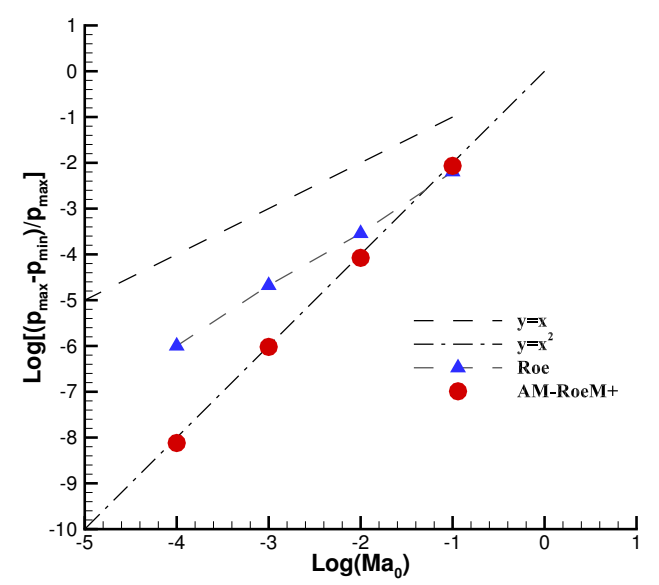

Figure 16: Pressure fluctuations with the inflow Mach number for the Roe scheme and the AM-RoeM+ scheme.

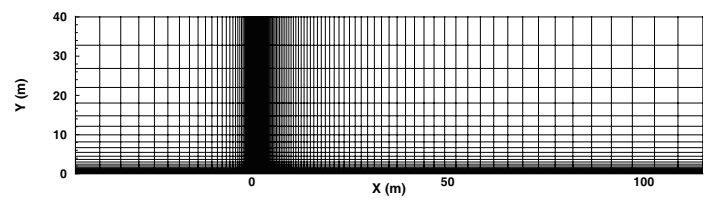

Figure 17: Computational mesh for the laminar boundary layer problem.

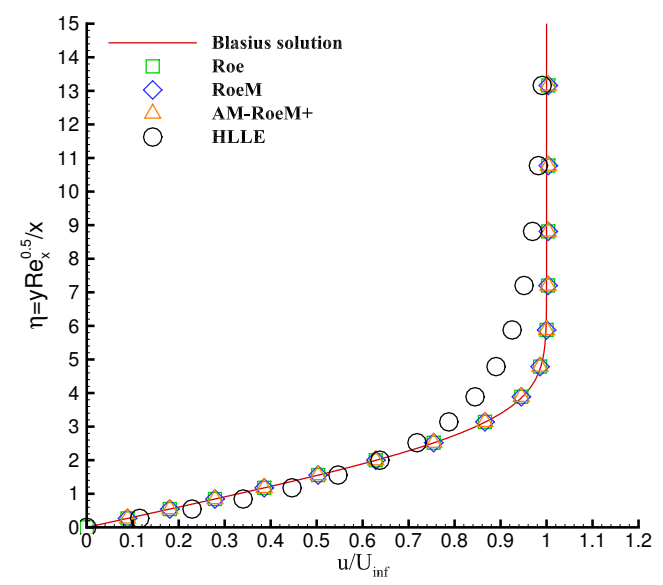

Figure 18: Non-dimensional velocity profile for the laminar boundary layer problem.

and the interval for $\mathrm{s}$ is divided into $n_{\xi}$ uniform sections.

The following flux functions are employed: Roe scheme with Harten's entropy fix (EFix) [19], RoeM and AM-RoeM+. To compute the left and right values at cell interfaces, 

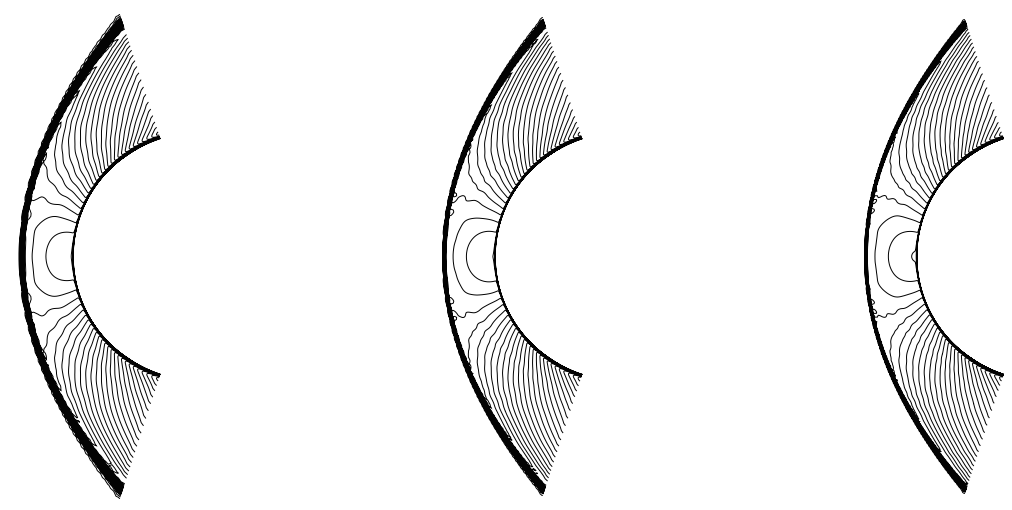

Figure 19: Comparison of density fields in Mach 8.1 viscous flow past a cylinder (Roe (E-Fix)). Left: $\left(n_{\xi}, n_{\eta}\right)=$ $(120,160)$; middle: $\left(n_{\xi}, n_{\eta}\right)=(180,240)$; and right: $\left(n_{\xi}, n_{\eta}\right)=(240,320)$. The range of the isolines is $[1.05 \leq$ $\left.\rho / \rho_{\text {inf }} \leq 6.9\right]$.
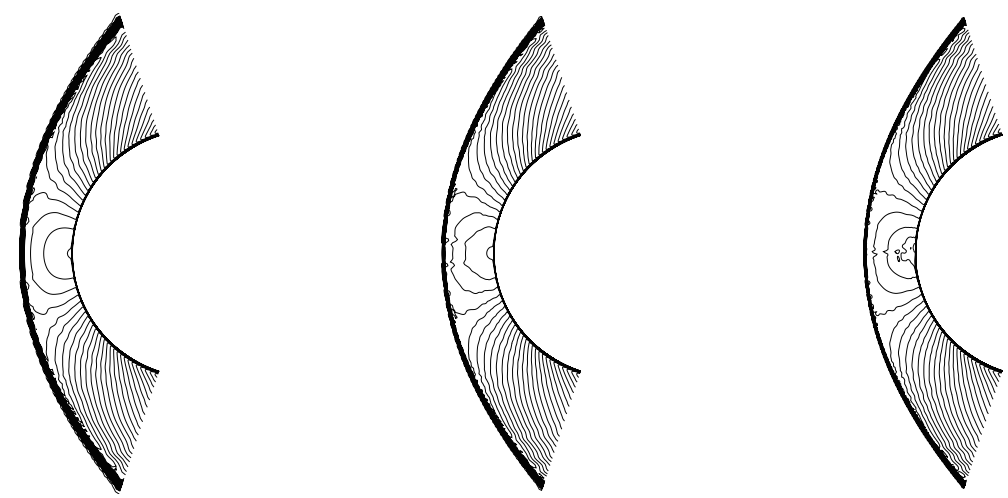

Figure 20: Comparison of density fields in Mach 8.1 viscous flow past a cylinder (RoeM). Left: $\left(n_{\xi}, n_{\eta}\right)=$ $(120,160)$; middle: $\left(n_{\xi}, n_{\eta}\right)=(180,240)$; and right: $\left(n_{\xi}, n_{\eta}\right)=(240,320)$. The range of the isolines is $[1.05 \leq$ $\left.\rho / \rho_{\text {inf }} \leq 6.9\right]$.

the 2nd order accurate MUSCL reconstruction with the min mod limiter is employed. The second-order central difference scheme is used to discrete the viscous term. For temporal discretization, the LU-SGS approach is employed. All the computations are conducted for 100,000 steps with $C F L=200$. The residuals defined as the L2-norm of density drop at least three orders of magnitude for all the cases. In Fig. 19-Fig. 21, the density fields of different flux functions for three meshes $\left(n_{\tilde{\xi}}, n_{\eta}\right)=(120,160),(180,240),(240,320)$ are depicted. As shown, the density fields computed by the Roe (E-Fix) flux function are overall good due to excess dissipation introduced by the entropy fix, but there are still some visible post-shock wrinkles. The density fields produced by the RoeM scheme exhibit shock anomalies, i.e., the carbuncle phenomenon and the post-shock wrinkles. These pathologies become even worse in finer meshes. However, the proposed AM-RoeM+ scheme 

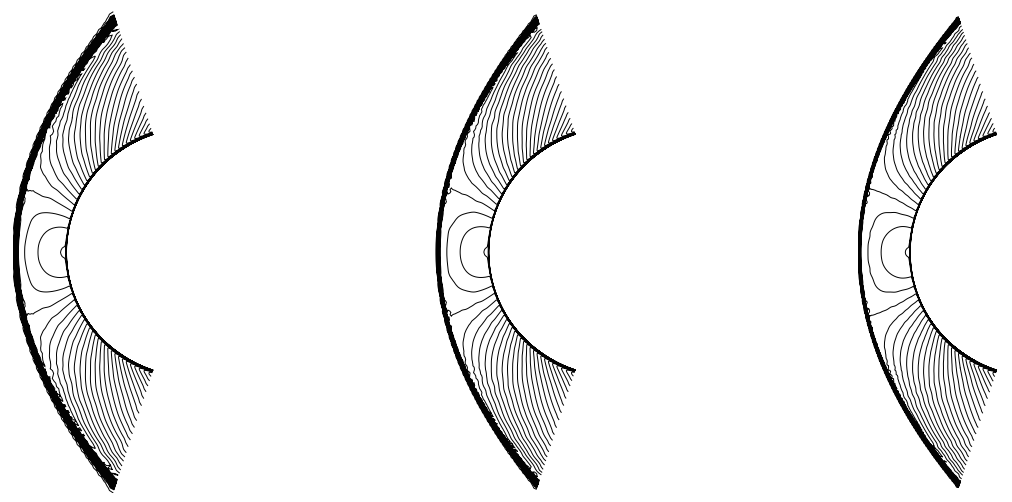

Figure 21: Comparison of density fields in Mach 8.1 viscous flow past a cylinder (AM-RoeM+). Left: $\left(n_{\tilde{\xi}}, n_{\eta}\right)=$ $(120,160)$; middle: $\left(n_{\xi}, n_{\eta}\right)=(180,240)$; and right: $\left(n_{\xi}, n_{\eta}\right)=(240,320)$. The range of the isolines is $[1.05 \leq$ $\left.\rho / \rho_{\text {inf }} \leq 6.9\right]$.

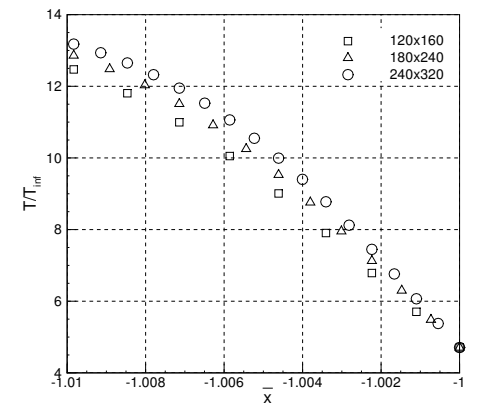

(a) Roe E-Fix

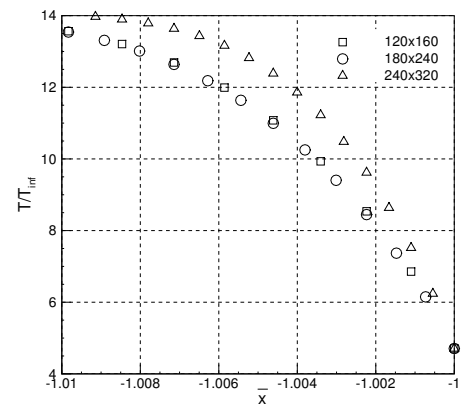

(b) RoeM

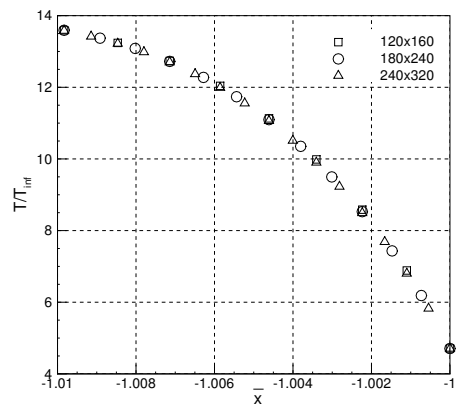

(c) AM-RoeM+

Figure 22: The temperature distribution in the boundary layer $(y=0)$.

still produces clear and symmetrical density fields and shock anomalies are not seen. The profiles of the nondimensional temperature $T / T_{\text {inf }}$ along $y=0$ in the boundary layer for different flux functions are demonstrated in Fig. 22. It can be seen that only the temperature profiles produced by the AM-RoeM+ scheme are nearly identical. In Fig. 23, we provide the profiles of the nondimensional heat flux $q / q_{\text {inf }}$ along the cylinder surface for different schemes. As shown, the results computed by the Roe (E-Fix) are mesh dependent and very inaccurate, the value of $q / q_{\text {inf }}$ at the stagnation point $(\theta=0)$ is underpredicted compared with the theoretical value $q / q_{\text {inf }}=2.46$, which is predicted by Fay-Riddell [61]. The RoeM scheme produces poor heat flux distributions due to shock anomalies. However, the results of the AM-RoeM+ scheme are nearly mesh independent and the value of $q / q_{\text {inf }}$ at the stagnation point is in good agreement with the theoretical value. The above results demonstrate that the proposed AM-RoeM+ is able to produce accurate and reliable results for hypersonic heating computations. 


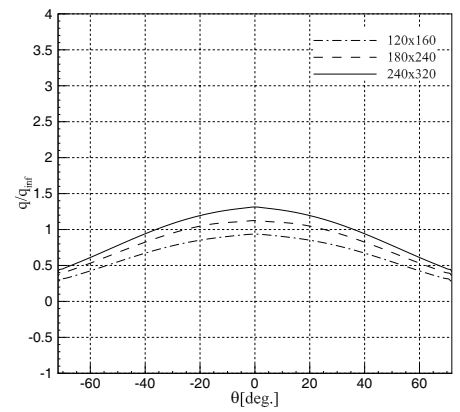

(a) Roe E-Fix

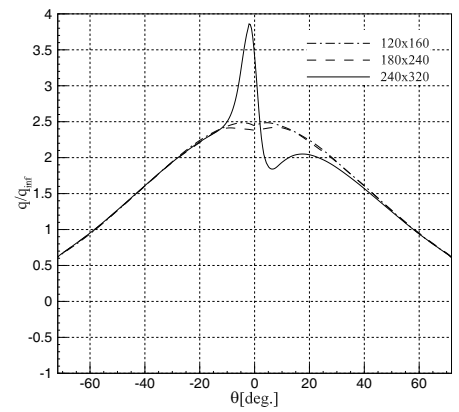

(b) RoeM

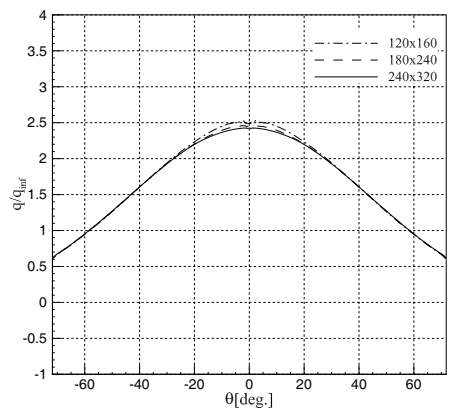

(c) AM-RoeM+

Figure 23: The distribution of the heat flux along the cylinder surface.

\section{Conclusions}

In this paper, we have developed a Roe-type scheme that is able to produce both accurate and robust solutions for all Mach number flow problems. In particular, by a linear perturbation analysis of the Roe-type scheme, we have shown that the shock instability of the original Roe method can be suppressed by a simple pressure perturbation-control technique without compromising its high resolution of linear degenerate waves. The main ingredient of this modification is based on the new understanding of the carbuncle mechanism that is first discussed in our previous work [14]. With an instability analysis of a simplified stationary normal shock problem, we are able to clarify that the pressure perturbation-control technique plays a part in reducing the perturbed mass flux behind the normal shock via limiting the propagation of pressure perturbations from inside the numerical shock structure to the downstream region. This makes it possible to cure the shock instability of Godunov-type schemes without resorting to any additional numerical dissipation on linear degenerate waves. We have also extended the Roe-type scheme to handle low Mach number problems accurately. Moreover, the resulting all Mach Roetype scheme does not involve any cut-off reference Mach number and user-defined parameters. Numerical results show that the proposed method is able to produce accurate and stable results across a large Mach number range. Finally, we remark that the pressure perturbation-control technique is also supposed to be applied to curing the shock instability of other Godunov-type schemes. Exploring such issues is left as a future work.

\section{Acknowledgements}

This work was supported by the National Natural Science Foundation of China (No. 11472004), the Foundation of Innovation of NUDT (No. B150106). 


\section{References}

[1] F. IsmaIL, Toward a Reliable Prediction of Shocks in Hypersonic Flow: Resolving Carbuncles with Entropy and Vorticity Control, University of Michigan, 2006.

[2] K. KITAMURA, P. ROE AND F. ISMAIL, Evaluation of Euler fluxes for hypersonic flow computations, AIAA J., 47 (2009), pp. 44-53.

[3] G. Candler, M. Barnhardt, T. Drayna, I. Nompelis, D. Peterson and P. SubBAREDDY, Unstructured Grid Approaches for Accurate Aeroheating Simulations, in: 18th AIAA Comput. Fluid Dyn. Conf., 2007.

[4] G. TU, X. ZhaO, M. MaO, J. Chen, X. Deng And H. LiU, Evaluation of Euler fluxes by a high-order CFD scheme: shock instability, Int. J. Comut. Fluid Dyn., 28 (2014), 171-186.

[5] K. KitAMURA AND E. SHIMA, Towards shock-stable and accurate hypersonic heating computations: A new pressure flux for AUSM-family schemes, J. Comput. Phys., 245 (2013), pp. 62-83.

[6] F. QU, C. YAN AND D. SUN, Investigation into the influences of the low speed's accuracy on the hypersonic heating computations, Int. Commun. Heat Mass Transf., 70 (2016), pp. 53-58.

[7] F. QU AND D. SUN, Investigation into the influences of the low-speed flows' accuracy on RANS simulations, Aerosp. Sci. Technol., 70 (2017), pp. 578-589.

[8] P. L. RoE, Approximate Riemann solvers, parameter vectors, and difference schemes, J. Comput. Phys., 43 (1981), pp. 357-372.

[9] A. HARTEN AND J. M. HYMAN, Self adjusting grid methods for one-dimensional hyperbolic conservation laws, J. Comput. Phys., 50 (1983), pp. 235-269.

[10] B. Einfeldt, C. D. MunZ, P. L. Roe And B. SJÖGReEn, On Godunov-type methods near low densities, J. Comput. Phys., 92 (1991), pp. 273-295.

[11] B. Parent, Positivity-preserving flux difference splitting schemes, J. Comput. Phys., 243 (2013), pp. 194-209.

[12] K. Peery And S. Imlay, Blunt-body flow simulations, in: 24th Jt. Propuls. Conf., American Institute of Aeronautics and Astronautics, 1988.

[13] Z. SHEN, W. YAN AND G. YUAN, A stability analysis of hybrid schemes to cure shock instability, Commun. Comput. Phys., 15 (2014), pp. 1320-1342.

[14] W. XIE, W. LI, H. LI, Z. TIAN AND S. PAN, On numerical instabilities of godunov-type schemes for strong shocks, J. Comput. Phys., 350 (2017), pp. 607-637.

[15] A. V. Rodionov, Artificial viscosity in Godunov-type schemes to cure the carbuncle phenomenon, J. Comput. Phys., 345 (2017), pp. 308-329.

[16] J. J. QUIRK, A contribution to the great Riemann solver debate, Int. J. Numer. Methods Fluids, 18 (1994), pp. 555-574.

[17] B. EINFELDT, On Godunov-type methods for gas dynamics, SIAM J. Numer. Anal., 25 (1988), pp. 294-318.

[18] G. Tchuen, F. Fogang, Y. Burtschell And P. WoAfo, A hybrid numerical method and its application to inviscid compressible flow problems, Comput. Phys. Commun., 185 (2014), pp. 479-488.

[19] A. HaRten, High resolution schemes for hyperbolic conservation laws, J. Comput. Phys., 49 (1983), pp. 357-393.

[20] H.-C. LIN, Dissipation additions to flux-difference splitting, J. Comput. Phys., 117 (1995), pp. 20-27.

[21] R. Sanders, E. Morano AND M.-C. Druguet, Multidimensional dissipation for upwind schemes: Stability and applications to gas dynamics, J. Comput. Phys., 145 (1998), pp. 511-537.

[22] Y.-X. REN, A robust shock-capturing scheme based on rotated Riemann solvers, Comput. Fluids, 
32 (2003), pp. 1379-1403.

[23] H. NiSHIKAWA AND K. KiTAMURA, Very simple, carbuncle-free, boundary-layer-resolving, rotated-hybrid Riemann solvers, J. Comput. Phys., 227 (2008), pp. 2560-2581.

[24] F. ZHANG, J. LIU, B. CHEN AND W. ZHONG, Evaluation of rotated upwind schemes for contact discontinuity and strong shock, Comput. Fluids, 134 (2016), pp. 11-22.

[25] F. ZHANG, J. LIU, B. CHEN AND W. ZHONG, A robust low-dissipation AUSM-family scheme for numerical shock stability on unstructured grids, Int. J. Numer. Methods Fluids, 84 (2017), pp. 135-151.

[26] E. Shima AND K. KITAMURA, Parameter-free simple low-dissipation AUSM-family scheme for all speeds, AIAA J., 49 (2011), pp. 1693-1709.

[27] S. F. DAVIS, Simplified second-order Godunov-type methods, SIAM J. Sci. Stat. Comput., 9 (1988), pp. $445-473$.

[28] S. S. KIM, C. KIM, O. H. RHO AND S. K. HonG, Cures for the shock instability: Development of a shock-stable Roe scheme, J. Comput. Phys., 185 (2003), pp. 342-374.

[29] M. S. Liou, Mass flux schemes and connection to shock instability, J. Comput. Phys., 160 (2000), pp. 623-648.

[30] M. DumbSer, J.-M. MoschetTA AND J. GRESSIER, A matrix stability analysis of the carbuncle phenomenon, J. Comput. Phys., 197 (2004), pp. 647-670.

[31] Z. SHEN, W. YAN AND G. YUAN, A robust HLLC-type riemann solver for strong shock, J. Comput. Phys., 309 (2016), pp. 185-206.

[32] H. GUillaRD AND C. VIOZAT, On the behaviour of upwind schemes in the low Mach number limit, Comput. Fluids, 28 (1999), pp. 63-86.

[33] H. GUILlard AND A. Murrone, On the behavior of upwind schemes in the low Mach number limit: II. Godunov type schemes, Comput. Fluids, 33 (2004), pp. 655-675.

[34] F. RIEPER, A low-Mach number fix for Roe's approximate Riemann solver, J. Comput. Phys., 230 (2011), pp. 5263-5287.

[35] X. LI AND C. GU, Mechanism of Roe-type schemes for all-speed flows and its application, Comput. Fluids, 86 (2013), pp. 56-70.

[36] S. Dellacherie, J. Jung, P. Omnes And P.-A. Raviart, Construction of modified Godunovtype schemes accurate at any Mach number for the compressible Euler system, Math. Model. Methods Appl. Sci., 26 (2016), pp. 2525-2615.

[37] B. Thornber, A. Mosedale, D. Drikakis, D. Youngs and R. J. R. Williams, an improved reconstruction method for compressible flows with low Mach number features, J. Comput. Phys., 227 (2008), pp. 4873-4894.

[38] SHU-SHENG CHEN, CHAO YAN AND XING-HAO XIANG, Effective low-Mach number improvement for upwind schemes, Comput. Math. Appl., 75 (2018), pp. 3737-3755.

[39] J. M. WEISS AND W. A. SMITH, Preconditioning applied to variable and constant density flows, AIAA J., 33 (1995), pp. 2050-2057.

[40] X. Li AND C. GU, An All-Speed Roe-type scheme and its asymptotic analysis of low Mach number behaviour, J. Comput. Phys., 227 (2008), pp. 5144-5159.

[41] X. LI, C. GU AND J. ZHONG XU, Development of Roe-type scheme for all-speed flows based on preconditioning method, Comput. Fluids, 38 (2009), pp. 810-817.

[42] K. Osswald, A. Siegmund, P. Birken, V. Hannemann And A. Meister, L2Roe: A low dissipation version of Roe's approximate Riemann solver for low Mach numbers, Int. J. Numer. Methods Fluids, 81 (2016), pp. 71-86.

[43] S. DellaCherIE, Analysis of Godunov type schemes applied to the compressible Euler system at low Mach number, J. Comput. Phys., 229 (2010), pp. 978-1016. 
[44] H. GuillaRd AND B. NKONGA, Chapter 8-On the behaviour of upwind schemes in the low Mach number limit: a review, in: R. Abgrall, C.-W. Shu (Eds.), Handb. Numer. Methods Hyperbolic Probl., Elsevier, (2017), pp. 203-231.

[45] P. L. ROE AND J. PIKE, Efficient construction and utilisation of approximate Riemann solutions, in: Proc. Sixth Int'L. Symp. Comput. Methods Appl. Sci. Eng., VI, North-Holland Publishing Co., Amsterdam, The Netherlands, The Netherlands, (1985), pp. 499-518.

[46] H. ZHU, X. DenG, M. MAO, H. LIU AND G. TU, Osher flux with entropy fix for twodimensional Euler equations, Adv. Appl. Math. Mech., 8 (2016), pp. 670-692.

[47] Barth, T. J., Some Notes on Shock-Resolving Flux Functions Part 1: Stationary Characteristics, NASA TM-101087, 1989.

[48] Y. CHAUvAT, J.-M. MOsChetTA AND J. GRESSIER, Shock wave numerical structure and the carbuncle phenomenon, Int. J. Numer. Methods Fluids, 47 (2005), pp. 903-909.

[49] F. IsMAIL AND P. L. ROE, Affordable, entropy-consistent euler flux functions ii: Entropy production at shocks, J. Comput. Phys., 228 (2009), pp. 5410-5436.

[50] W. XIE, W. LI, H. LI, S. PAN AND Z. TIAN, On the accuracy and robustness of a new flux splitting method, Acta Phys. Sin., 64 (2015), 024702.

[51] J. GRESSIER AND J.-M. MOSCHETTA, Robustness versus accuracy in shock-wave computations, Int. J. Numer. Methods Fluids, 33 (2000), pp. 313-332.

[52] G. A. SoD, Numerical Methods in Fluid Dynamics: Initial and Initial Boundary-Value Problems, No. 1 in Numerical Methods in Fluid Dynamics, Cambridge University Press, 1985.

[53] T. OHWADA, R. ADACHI, K. XU AND J. LUO, On the remedy against shock anomalies in kinetic schemes, J. Comput. Phys., 255 (2013), pp. 106-129.

[54] M.-S. LIOU, A sequel to AUSM: AUSM+, J. Comput. Phys., 129 (1996), pp. 364-382.

[55] M.-S. LIOU, A sequel to AUSM, Part II: AUSM+-up for all speeds, J. Comput. Phys., 214 (2006), pp. 137-170.

[56] E. F. TORO, Riemann Solvers and Numerical Methods for Fluid Dynamics: A Practical Introduction, Springer Berlin Heidelberg, 2013.

[57] P. WOODWARD AND P. COlella, The numerical simulation of two-dimensional fluid flow with strong shocks, J. Comput. Phys., 54 (1984), pp. 115-173.

[58] C.-W. SHU AND S. OSHER, Efficient implementation of essentially non-oscillatory shockcapturing schemes, J. Comput. Phys., 77 (1988), pp. 439-471.

[59] P. M. GRESHO AND S. T. CHAN, On the theory of semi-implicit projection methods for viscous incompressible flow and its implementation via a finite element method that also introduces a nearly consistent mass matrix: part 2: Implementation, Int. J. Numer. Methods Fluids, 11 (1990), pp. 621-659.

[60] K. KitAmura, E. Shima, Y. NAKAmURA AND P. ROE, Evaluation of Euler fluxes for hypersonic heating computations, AIAA J., 48 (2010), pp. 763-776.

[61] J. A. FAY AND F. R. RIDDELL, Theory of stagnation point heat transfer in dissociated air, J. Aeronaut. Sci., 25 (1958), pp. 73-85. 